\title{
Existence of Solutions via a New Variational Principle for Nonlocal Semilinear Elliptic Equations
}

\section{K.L. Wong}

A thesis submitted to

the Faculty of Graduate and Postdoctoral Affairs

in partial fulfillment of the requirements for the degree of

\author{
Master of Science \\ in \\ Mathematics
}

School of Mathematics and Statistics

Ottawa-Carleton Institute for Mathematics and Statistics

Carleton University

Ottawa, Ontario, Canada

\author{
(C) Copyright \\ 2018, K.L. Wong
}




\section{Abstract}

The aim of this thesis is to prove the existence of a weak solution for semilinear fractional elliptic equations given by

$$
\begin{cases}(-\Delta)^{s} u=|u|^{p-2} u+f(x), & x \in \Omega, \\ u=0, & x \in \mathbb{R}^{n} \backslash \Omega,\end{cases}
$$

where $(-\Delta)^{s}$ denotes the fractional Laplace operator with $s \in(0,1], n>2 s$ where $n \in \mathbb{Z}, \Omega$ is an open bounded domain in $\mathbb{R}^{n}$ with $C^{2}$-boundary and $f \in L^{2}(\Omega)$. Note that when $s=1$, we obtain the classical semilinear elliptic equation given by

$$
\begin{cases}-\Delta u=|u|^{p-2} u+f(x), & x \in \Omega, \\ u=0, & x \in \partial \Omega .\end{cases}
$$

In the classical case when $s=1$, the result is known for $p$ less than the critical Sobolev exponent $2^{*}=(2 n) /(n-2)$ for which the Sobolev embedding $H^{1}(\Omega) \hookrightarrow L^{p}(\Omega)$ is compact. In this thesis, we are interested in extending this result for $s \in(0,1]$ to $p$ greater than the critical Sobolev exponent where the compact embedding fails to hold. We shall make use of a new variational principle presented in [17] that allows one to deal with problems well beyond the compact structure. 


\section{Acknowledgements}

I would like to take this opportunity to express my sincerest gratitude to my supervisor, Dr. Abbas Momeni for his constant support, guidence, and undying patience. His immense knowledge in the subject has enlightened me in many ways possible and all the valuable time spent learning with him has been cherished deeply. In addition, thank you for constantly believing in me.

I would like to extend my thanks to the faculty and staff of the School of Mathematics and Statistics at Carleton University for their help and support throughout my M.Sc studies.

Furthermore, I would like to extend my thanks to my friends and colleagues who has taken part of my journey as a graduate student.

Finally, I would like to expess my deepest gratitude to my family for their endless belief and encouragement on my journey towards my academic career in mathematics. Without their advice, support, and unconditional love, I would not be where I am today. I am constantly being inspired by each and every one of my family members as it allows me to better myself in all ways possible. Thank you all for being the biggest support team I have in my life. 


\section{Contents}

Title Page $\quad$ i

Abstract

Acknowledgements

Table of Contents

1 Introduction 1

2 Preliminaries $\quad 6$

2.1 Sobolev Spaces . . . . . . . . . . . . . . . . 6

2.1.1 The Lebesgue Space $L^{p}(\Omega) \ldots \ldots$. . . . . . . . . . . 7

2.1.2 The Sobolev Space $W^{k, p}(\Omega)$. . . . . . . . . . . . . . . . 10

2.2 Fractional Sobolev Spaces . . . . . . . . . . . . . . 13

2.2.1 The Fractional Laplace Operator . . . . . . . . . . . . . . 13

2.2.2 The Fractional Sobolev Space $W^{s, p}$. . . . . . . . . . . . . 15

2.2.3 The Fractional Sobolev-Type Space $X^{s} \ldots \ldots$. . . . . . . . 17

2.3 Embedding Properties . . . . . . . . . . . . . . . 21

2.3.1 The Lebesgue Space $L^{p}(\Omega) \ldots \ldots$. . . . . . . . . . . . . . . 21

2.3.2 The Sobolev Space $W^{k, p}(\Omega)$. . . . . . . . . . . . 22

2.3.3 The Fractional Sobolev Space $W^{s, p}(\Omega)$. . . . . . . . . . . 24

2.3.4 The Fractional Sobolev-type Space $X_{0}^{s}(\Omega)$. . . . . . . . . 26 
2.4 Regularity Theory . . . . . . . . . . . . . . . . 27

2.5 Convex Analysis . . . . . . . . . . . . . . . . . . . . 31

3 A New Variational Principle 34

4 Nonhomogeneous Local and Nonlocal Semilinear Elliptic Equa$\begin{array}{ll}\text { tions } & 38\end{array}$

4.1 Semilinear Elliptic Equation Involving the Classical Laplacian . . 39

4.2 Semilinear Elliptic Equation Involving the Fractional Laplacian 50

$\begin{array}{ll}\text { Bibliography } & 65\end{array}$ 


\section{Chapter 1}

\section{Introduction}

In this thesis, we study the existence of a solution for the following semilinear elliptic problem given by

$$
\begin{cases}-\Delta u=|u|^{p-2} u+f(x), & x \in \Omega, \\ u=0, & x \in \partial \Omega,\end{cases}
$$

where $\Omega$ is an open bounded domain in $\mathbb{R}^{n}$ with $C^{2}$-boundary and $f \in L^{2}(\Omega)$.

There have been numerous studies on problems of type (1.1) dealing with subcritical values of $p$ i.e., when $p$ is less than the critical Sobolev exponent $2^{*}=(2 n) /(n-2)$. In [4], Bahri showed that for each $k \in \mathbb{N}$, there exists an open and dense subset $\Lambda \subset L^{2}(\Omega)$ such that there are $k$ distinct solutions for each $f \in \Lambda$. When $p \geq 2^{*}$, the compact embedding $H^{1}(\Omega) \hookrightarrow L^{p}(\Omega)$ fails to hold. This causes difficulties when solving problems of type (1.1) as we can no longer apply standard variational methods in a straight-forward manner. However, in [32], for $p=2^{*}$, using the methods of minimization and minimax principles of mountain pass-type presented in [2], Tarantello showed that for suitable functions $f \in L^{2}(\Omega)$, one can produce critical values of the energy functional associated with (1.1) given by

$$
I[u]=\frac{1}{2} \int_{\Omega}|\nabla u|^{2}-\frac{1}{p} \int_{\Omega}|u|^{p}-\int_{\Omega} f u, \quad u \in H_{0}^{1}(\Omega),
$$


even though the Palais-Smale condition may fail to hold.

In addition, there have been several studies on problems of type (1.1) dealing with certain shapes of the domain $\Omega$, in particular, the problem given by

$$
\begin{cases}\Delta u+|u|^{p-2} u=0, & \text { in } \Omega, \\ u \neq 0, & \text { in } \Omega, \\ u=0, & \text { on } \partial \Omega,\end{cases}
$$

where $\Omega$ is a bounded domain in $\mathbb{R}^{n}$ with $n \geq 3$.

When $p<2^{*}$, the existence of positive and nodal (sign-changing) solutions of (1.2) does not depend on the shape of $\Omega$. However, when $p \geq 2^{*}$, the existence of solutions of problem (1.2) depends solely on the shape of $\Omega$. In [23], Pohozaev showed that no solutions exist if $\Omega$ is star-shaped. On the contrary, if $\Omega$ is an annulus, Kazdan and Warner have pointed out in [16] the existence of a radial solution. For some contractible domains that are not star-shaped, multiplicity of positive solutions for $p=2^{*}$ are established in [10,21, 22]. When $p>2^{*}$, Passaseo presented in $[19,20]$ multiplicity of nodal solutions.

We shall establish the existence of solutions to (1.1) where the value of $p$ can be extended past the critical Sobolev exponent $2^{*}$. The tools we will need for proving this result will be a new variational principle presented in [17] in combination with the elliptic regularity theory given in [14].

In addition to the problem presented in (1.1), we are also interested in the existence of a solution for the following nonlocal semilinear elliptic problem

$$
\begin{cases}(-\Delta)^{s} u=|u|^{p-2} u+f(x), & x \in \Omega, \\ u=0, & x \in \mathbb{R}^{n} \backslash \Omega,\end{cases}
$$

where $s \in(0,1), n>2 s, \Omega$ is an open bounded domain in $\mathbb{R}^{n}$ with $C^{2}$-boundary, $f \in L^{2}(\Omega)$, and $(-\Delta)^{s}$ denotes the fractional Laplace operator defined by

$$
(-\Delta)^{s} u(x)=-\frac{1}{2} C(n, s) \int_{\mathbb{R}^{n}} \frac{u(x+y)+u(x-y)-2 u(x)}{|y|^{n+2 s}} d y, \quad x \in \mathbb{R}^{n} .
$$


In recent years, there has been a rising interest in the study of fractional Laplace equations. Consider the following nonlocal subcritical problem

$$
\begin{cases}(-\Delta)^{s} u-\lambda u=|u|^{p-2} u+f, & x \in \Omega, \\ u=0, & x \in \mathbb{R}^{n} \backslash \Omega,\end{cases}
$$

where $\Omega$ is an open bounded subset of $\mathbb{R}^{n}$ with Lipschitz boundary and $\lambda \in \mathbb{R}$. In [28], Servadei adapted the standard variational methods used in the study of the classical Laplace equation with subcritical nonlinearities to the nonlocal framework and obtained that for $p \in\left(2,2_{s}^{*}-(2 s) /(n-2 s)\right)$, where $2_{s}^{*}=(2 n) /(n-$ $2 s$ ) is the fractional critical Sobolev exponent, problem (1.5) admits infinitely many weak solutions $u_{k} \in H^{s}\left(\mathbb{R}^{n}\right)$ with $u_{k}=0$ a.e. in $\mathbb{R}^{n} \backslash \Omega$ and its Sobolev norm goes to infinity as $k \rightarrow \infty$, i.e.,

$$
\int_{\mathbb{R}^{n} \times \mathbb{R}^{n}} \frac{\left|u_{k}(x)-u_{k}(y)\right|^{2}}{|x-y|^{n+2 s}} d x d y \rightarrow \infty \text { as } k \rightarrow \infty .
$$

For the case when $f \equiv 0$, Servadei and Valdinoci presented in [30] the existence of a non-trivial weak solution for different values of $\lambda$.

Similar to the local problems visited previously, when $p \geq 2{ }_{s}^{*}$, we come across some difficulty due to the lack of compactness. Thus, the adaptation of standard variational methods used in the subcritical problem fail to hold. However, in [29], Servadei and Valdinoci applied variants of classical variational methods provided in [2] to show that the following general nonlocal problem

$$
\begin{cases}\mathcal{L}_{K} u+\lambda u+|u|^{2^{*}-2} u+f(x, u)=0, & \text { in } \Omega, \\ u=0, & \text { in } \mathbb{R}^{n} \backslash \Omega\end{cases}
$$

admits non-trivial weak solutions where $f$ is subcritical, $\lambda>0$, and $\mathcal{L}_{K}$ is the integrodifferential operator defined by

$$
\mathcal{L}_{K} u(x)=\int_{\mathbb{R}^{n}}(u(x+y)+u(x-y)-2 u(x)) K(y) d y, \quad x \in \mathbb{R}^{n}
$$

with the function $K: \mathbb{R}^{n} \backslash\{0\} \rightarrow(0, \infty)$ such that 
(i) $m K \in L^{1}\left(\mathbb{R}^{n}\right)$, where $m(x):=\min \left\{|x|^{2}, 1\right\}$ and;

(ii) there exists $\theta>0$ such that $K(x) \geq \theta|x|^{-(n+2 s)}$, for any $x \in \mathbb{R}^{n} \backslash\{0\}$.

For the case where $K$ is given by $K(x)=|x|^{-(n+2 s)}$, we get the fractional Laplace operator $(-\Delta)^{s}$ defined in (1.4). Thus, in [29] and references therein, the authors were devoted to the study of the existence of solutions to the critical problem

$$
\begin{cases}(-\Delta)^{s} u-\lambda u=|u|^{2^{*}-2} u, & \text { in } \Omega, \\ u=0, & \text { in } \mathbb{R}^{n} \backslash \Omega\end{cases}
$$

Analogous to problem (1.2), we visit the counterpart of problem (1.2), i.e.,

$$
\begin{cases}(-\Delta)^{s} u=|u|^{p-2} u, & \text { in } \Omega, \\ u \neq \equiv 0, & \text { in } \Omega, \\ u=0, & \text { in } \mathbb{R}^{n} \backslash \Omega .\end{cases}
$$

We see that similar results obtained for problem (1.2) hold in the nonlocal framework in terms of the shape of $\Omega$. To briefly review the dependence of the topology of the domain $\Omega$, we list the results below:

(i) If the domain $\Omega$ is star-shaped, then (1.6) admits trivial solutions for supercritical nonlinearities (see [26]);

(ii) If the domain $\Omega$ is of annular type, then for $p=2_{s}^{*}$, (1.6) admits a positive solution provided that the hole of the annulus is sufficiently small in size (see [27]) and;

(iii) If $\Omega$ is a suitable contractible domain, then for $p=2_{s}^{*}$, (1.6) admits a positive solution (see [18]).

Note that when $p<2_{s}^{*}$, the existence of solutions do not depend on the topology of $\Omega$. 
Analogous to the local problem, we shall establish the existence of solutions to $(1.3)$ where $p$ can be extended past the fractional critical Sobolev exponent $2_{s}^{*}$. To prove the existence of a weak solution to problem (1.3), we once again apply the new variational principle given in [17] along with the $L^{q}$-regularity of weak solutions provided in [6].

The thesis is presented in the following manner. We review in Chapter 2 some basic definitions and preliminary results in which we will utilize throughout the thesis. We begin by reviewing the definition of Lebesgue spaces along with some simple yet important inequalities that we will be using in the proof of our results. Afterwards, we jump to the topic of Sobolev spaces which encompasses the standard Sobolev space $W^{k, p}$, the fractional Sobolev space $W^{s, p}$, and the fractional Sobolev-type space $X^{s}$. Then we devote a section of the chapter to the continuous and compact embeddings of these Sobolev spaces. We conclude the chapter with a section on the elliptic regularity and the $L^{q}$-regularity results which play important roles in proving our results followed by a section dedicated to some essential definitions on convex analysis for lower semi-continuous functions.

In Chapter 3, we begin with a general discussion on variational problems followed by the new variational principle which is the focal point of the thesis. Finally in Chapter 4, we present the proof of our results of the thesis. We begin by looking at the local semilinear elliptic equation with values of $p$ exceeding the critical Sobolev exponent followed by the nonlocal semilinear elliptic problem. 


\section{Chapter 2}

\section{Preliminaries}

In this chapter, we review some definitions and basic properties that we find useful throughout the thesis. We begin in Section 2.1 with the standard Sobolev spaces. In Section 2.2, when standard Sobolev spaces extend to fractional parameters, we introduce the fractional Sobolev spaces that allows one to deal with nonlocal problems. We present continuous and compact embeddings in Section 2.3 for both the standard Sobolev spaces and the fractional Sobolev spaces followed by some regularity results in Section 2.4. We end the chapter with some useful concepts in convex analysis for lower semi-continuous functions.

\subsection{Sobolev Spaces}

In this section, we review the theory of Sobolev spaces. The significance of Sobolev spaces is to deal with problems in mathematical physics and variational calculus in which it is not sufficient to find classical solutions of differential equations. We weaken this concept by introducing the notion of weak derivatives and work in these weakened spaces. Before we jump to the definition and properties of Sobolev spaces, we review some important facts of Lebesgue spaces which will be beneficial to the readers in following along with the notion of Sobolev spaces. 


\subsubsection{The Lebesgue Space $L^{p}(\Omega)$}

Throughout this subsection, we will denote $\Omega$ to be a bounded domain in $\mathbb{R}^{n}$. We review some definitions and foundations in functional analysis from [13] before we introduce Lebesgue spaces. Consider the real linear space $V$ and recall the definition of a norm on $V$ as follows.

Definition 2.1.1 (Norm). A mapping $\|\cdot\|: V \rightarrow[0, \infty)$ is called a norm if

(i) $\|u\|>0$ for all $u \in V,\|u\|=0$ if and only if $u=0$.

(ii) $\|\lambda u\|=|\lambda|\|u\|$ for all $u, \in V, \lambda \in \mathbb{R}$.

(iii) $\|u+v\| \leq\|u\|+\|v\|$ for all $u, v \in V$.

From this point on, we consider $V$ to be a normed linear space over $\mathbb{R}$.

Definition 2.1.2 (Convergence). We say a sequence $\left\{u_{j}\right\}_{j=1}^{\infty} \subset V$ converges to $u \in V$, written

$$
u_{j} \rightarrow u
$$

if

$$
\lim _{j \rightarrow \infty}\left\|u_{j}-u\right\|=0
$$

Definition 2.1.3 (Cauchy sequence). A sequence $\left\{u_{j}\right\}_{j=1}^{\infty} \subset V$ is called a Cauchy sequence provided for each $\epsilon>0$ there exists $N>0$ such that

$$
\left\|u_{j}-u_{k}\right\|<\epsilon \text { for all } j, k \geq N \text {. }
$$

Recall that every convergent sequence is Cauchy however, it is not necessarily true that every Cauchy sequence converges. If every Cauchy sequence converges, then we say that a normed linear space $V$ is complete.

Definition 2.1.4 (Banach space). A Banach space $V$ is a complete, normed linear space. 
Definition 2.1.5 (Dual space). Let $V$ be a Banach space. We call a bounded linear operator $u^{*}: V \rightarrow \mathbb{R} a$ bounded linear functional. We define the dual space $V^{*}$ of $V$ by

$$
V^{*}=\{\text { all bounded linear functionals on } V\} \text {. }
$$

Recall that if $u \in V, u^{*} \in V^{*}$, then we denote the real number $u^{*}(u)$ by

$$
u^{*}(u)=\left\langle u^{*}, u\right\rangle
$$

where $\langle\cdot, \cdot\rangle$ denotes the pairing of $V^{*}$ and $V$.

Definition 2.1.6 (Reflexive Banach space). Let $V$ be a Banach space. Suppose $\left(V^{*}\right)^{*}=V$ i.e., for all $u^{* *} \in\left(V^{*}\right)^{*}$, there exists $u \in V$ such that

$$
\left\langle u^{* *}, u^{*}\right\rangle=\left\langle u^{*}, u\right\rangle .
$$

Then we call $V$ a reflexive Banach space.

Now we can define the notion of weak convergence and weak compactness. Let $V$ be a normed linear space. We say a sequence $\left\{u_{j}\right\}_{j=1}^{\infty} \subset V$ converges weakly to $u \in V$, written

$$
u_{j} \rightarrow u,
$$

if

$$
\left\langle u^{*}, u_{j}\right\rangle \rightarrow\left\langle u^{*}, u\right\rangle,
$$

for all $u^{*} \in V^{*}$.

Theorem 2.1.7 (Weak compactness). A bounded sequence in a reflexive Banach space contains a weakly convergence subsequence (see [14] on Pg. 85).

The interested reader can refer to $[14,35]$ for the proof of Theorem 2.1.7. Now let us consider a real linear space $H$ and recall the definition of an inner product on $V$ as follows. 
Definition 2.1.8 (Inner product). A mapping $(\cdot, \cdot): H \times H \rightarrow \mathbb{R}$ is called an inner product if

(i) $(u, v)=(v, u)$ for all $u, v \in H$,

(ii) the mapping $u \mapsto(u, v)$ is linear for each $v \in H$,

(iii) $(u, u) \geq 0$ for all $u \in H,(u, u)=0$ if and only if $u=0$.

Note that if $(\cdot, \cdot)$ is an inner product, then we say that the inner product generates a norm given by

$$
\|u\|:=\sqrt{(u, u)}
$$

for all $u \in H$.

Definition 2.1.9 (Hilbert space). A Hilbert space $H$ is a Banach space endowed with an inner product which generates a norm.

Now we can take a look at Lebesgue spaces. For $1 \leq p<\infty$, we define the Lebesgue space $L^{p}(\Omega)$ to be the class of all measurable functions $u$ defined on $\Omega$ such that

$$
\int_{\Omega}|u(x)|^{p} d x<\infty
$$

Equivalently, measurable functions $u$ on $\Omega$ in the space $L^{p}(\Omega)$ are $p$-integrable. We define the norm in $L^{p}(\Omega)$ by

$$
\|u\|_{L^{p}(\Omega)}=\left(\int_{\Omega}|u(x)|^{p} d x\right)^{\frac{1}{p}}
$$

provided $1 \leq p<\infty$

For $p=\infty$, we define the space $L^{\infty}(\Omega)$ to be the linear space of all functions $u$ that are essentially bounded on $\Omega$. We define the norm in $L^{\infty}(\Omega)$ by

$$
\|u\|_{L^{\infty}(\Omega)}=\operatorname{ess}_{x \in \Omega} \sup |u(x)|
$$

where $\operatorname{ess}_{\sup }|u(x)|$ defines a measurable function $u$ on $\Omega$ to be essentially bounded on $\Omega$. 
Remark 2.1.10. We define the space $L_{l o c}^{p}(\Omega)$ of locally integrable functions by

$$
L_{\text {loc }}^{p}(\Omega):=\left\{u: \Omega \rightarrow \mathbb{R}:\|u\|_{L^{p}\left(\Omega^{\prime}\right)}<\infty \text { for every compact subset } \Omega^{\prime} \text { of } \Omega\right\} .
$$

We end this subsection with two standard inequalities which are important for the study of our problems. We present these inequalities without proof.

Theorem 2.1.11 (Hölder's Inequality). Let $1<p<\infty$ and let $p^{\prime}$ denote the conjugate exponent given by

$$
p^{\prime}=\frac{p}{p-1}, \quad \text { that is } \quad \frac{1}{p}+\frac{1}{p^{\prime}}=1,
$$

which also satisfies $1<p^{\prime}<1$. If $u \in L^{p}(\Omega)$ and $v \in L^{p^{\prime}}(\Omega)$, then $u v \in L^{1}(\Omega)$, and

$$
\int_{\Omega}|u(x) v(x)| d x \leq\|u\|_{L^{p}(\Omega)}\|v\|_{L^{p^{\prime}}(\Omega)} .
$$

Equality holds if and only if $|u(x)|^{p}$ and $|v(x)|^{p^{\prime}}$ are proportional almost everywhere (a.e.) in $\Omega$ (see [1] Theorem 2.4).

Theorem 2.1.12 (An Interpolation Inequality). Let $1 \leq p<q<r$, so that

$$
\frac{1}{q}=\frac{\theta}{p}+\frac{1-\theta}{r}
$$

for some $\theta$ satisfying $0<\theta<1$. If $u \in L^{p}(\Omega) \cap L^{r}(\Omega)$, then $u \in L^{q}(\Omega)$ and

$$
\|u\|_{L^{q}(\Omega)} \leq\|u\|_{L^{p}(\Omega)}^{\theta}\|u\|_{L^{r}(\Omega)}^{1-\theta}
$$

(see [1] Theorem 2.11).

\subsubsection{The Sobolev Space $W^{k, p}(\Omega)$}

Before we state the definition of the Sobolev space $W^{k, p}(\Omega)$, we need to introduce the definition of weak derivatives. 
Let $C_{0}^{\infty}(\Omega)$ be the space of infinitely differentiable functions $\phi: \Omega \rightarrow \mathbb{R}$ with compact support in $\Omega$. Let $\alpha=\left(\alpha_{1}, \ldots, \alpha_{n}\right)$ where $\alpha_{j}$ is a nonnegative integer for $j=1, \ldots, n$. We call $\alpha$ a multi-index and we define the length of $\alpha$ by

$$
|\alpha|=\sum_{j=1}^{n} \alpha_{j}=\alpha_{1}+\cdots+\alpha_{n} .
$$

Let $\alpha$ be a multi-index. Then we define $D^{\alpha}$ by

$$
D^{\alpha} u(x):=\frac{\partial^{|\alpha|} u(x)}{\partial x_{1}^{\alpha_{1}} \cdots \partial x_{n}^{\alpha_{n}}}=\partial_{x_{1}}^{\alpha_{1}} \cdots \partial_{x_{n}}^{\alpha_{n}} u .
$$

Definition 2.1.13 (Weak Derivatives). Suppose $u, v \in L_{l o c}^{1}(\Omega)$, and $\alpha$ is a multiindex. We say that $v$ is the $\alpha^{\text {th }}$-weak partial derivative of $u$, written

$$
D^{\alpha} u=v,
$$

provided

$$
\int_{\Omega} u D^{\alpha} \phi d x=(-1)^{|\alpha|} \int_{\Omega} v \phi d x
$$

for all test functions $\phi \in C_{0}^{\infty}(\Omega)$ (see [13] on Pg. 242).

The notion of weak derivatives allow us to introduce other kinds of spaces which contain less smooth functions. This leads us to the definition of the Sobolev space $W^{k, p}(\Omega)$.

Definition 2.1.14. Fix $1 \leq p \leq \infty$ and let $k$ be a nonnegative integer. The Sobolev space

$$
W^{k, p}(\Omega)
$$

consists of all locally summable functions $u: \Omega \rightarrow \mathbb{R}$ such that for each multiindex $\alpha$ with $|\alpha| \leq k, D^{\alpha} u$ exists in the weak sense and belongs to $L^{p}(\Omega)$ (see [13] on Pg. 244).

Definition 2.1.15. If $u \in W^{k, p}(\Omega)$, we define its norm to be

$$
\|u\|_{W^{k, p}(\Omega)}:= \begin{cases}\left(\sum_{|\alpha| \leq k} \int_{\Omega}\left|D^{\alpha} u\right|^{p} d x\right)^{\frac{1}{p}} & (1 \leq p<\infty), \\ \sum_{|\alpha| \leq k} \operatorname{ess}_{\sup }\left|D^{\alpha} u\right| & (p=\infty)\end{cases}
$$

(see [13] on Pg. 245). 
Definition 2.1.16. Let $\left\{u_{j}\right\}_{j=1}^{\infty}, u \in W^{k, p}(\Omega)$. We say that $u_{j}$ converges to $u$ in $W^{k, p}(\Omega)$, written

$$
u_{j} \rightarrow u \quad \text { in } W^{k, p}(\Omega)
$$

provided

$$
\lim _{j \rightarrow \infty}\left\|u_{j}-u\right\|_{W^{k, p}(\Omega)}=0
$$

(see [13] on Pg. 245).

Theorem 2.1.17. For each nonnegative integer $k$ and $1 \leq p \leq \infty$, the Sobolev space $W^{k, p}(\Omega)$ is a Banach space (see [13] on Pg. 249).

If $p=2$, then we denote $H^{k}(\Omega)=W^{k, 2}(\Omega)$, where $k$ is a nonnegative integer. In fact, $H^{k}(\Omega)$ is a Hilbert space. Finally, we end this subsection with the definition of $W_{0}^{k, p}(\Omega)$.

Definition 2.1.18. We denote by

$$
W_{0}^{k, p}(\Omega)
$$

the closure of $C_{0}^{\infty}(\Omega)$ in $W^{k, p}(\Omega)$ (see [13] on Pg. 245).

In other words, we can view $W_{0}^{k, p}(\Omega)$ to be the space consisting of functions $u \in W^{k, p}(\Omega)$ such that

$$
D^{\alpha} u=0 \text { on } \partial \Omega \text { for all }|\alpha| \leq k-1
$$

With this definition, we can define the dual space $W^{-k, p}(\Omega)$ of $W_{0}^{k, q}(\Omega)$ by

$$
W^{-k, p}(\Omega):=\left(W_{0}^{k, q}(\Omega)\right)^{\prime},
$$

for nonnegative integers $k$ where $\frac{1}{p}+\frac{1}{q}=1$. Once again for $p=2$, we denote $H_{0}^{k}(\Omega)=W_{0}^{k, 2}(\Omega)$, where $k$ is a nonnegative integer. 


\subsection{Fractional Sobolev Spaces}

In this section, we introduce the fractional Laplacian $(-\Delta)^{s}$ of order $2 s$ along with fractional Sobolev spaces. The fractional Laplacian $(-\Delta)^{s}$ is a nonlocal operator as we shall see later in this section. In order to work with this nonlocal operator, we need a suitable space which is the fractional Sobolev space.

\subsubsection{The Fractional Laplace Operator}

We present in this subsection the definition of the fractional Laplace operator $(-\Delta)^{s}$. Throughout this subsection, we refer to the following definitions and properties given in [11] and [15]. Observe when $s=1$, we obtain the standard Laplace operator $-\Delta$ and when $s=0$, we obtain the identity operator. We are particularly interested in the case where $s \in(0,1)$.

Before we introduce the definition of the fractional Laplace operator, we consider the Schwartz space of rapidly decaying functions defined by

$$
\mathcal{S}:=\mathcal{S}\left(\mathbb{R}^{n}\right):=\left\{u \in C^{\infty}\left(\mathbb{R}^{n}\right): \sup _{x \in \mathbb{R}^{n}}\left|x^{\alpha}\left(D^{\beta} u\right)(x)\right|<\infty\right\},
$$

where $\alpha \geq 0$ and $\beta \geq 0$ are multi-indices and $C^{\infty}\left(\mathbb{R}^{n}\right)$ is the space of smooth functions from $\mathbb{R}^{n}$ to $\mathbb{C}$.

Remark 2.2.1. We have the following inclusions,

$$
C_{0}^{\infty}\left(\mathbb{R}^{n}\right) \subset \mathcal{S}\left(\mathbb{R}^{n}\right) \subset C^{\infty}\left(\mathbb{R}^{n}\right)
$$

By definition, $\mathcal{S}\left(\mathbb{R}^{n}\right) \subset C^{\infty}\left(\mathbb{R}^{n}\right)$. To see that $C_{0}^{\infty}\left(\mathbb{R}^{n}\right) \subset \mathcal{S}\left(\mathbb{R}^{n}\right)$, consider the function

$$
u(x)=e^{\frac{-|x|^{2}}{2}}, \text { for } x \in \mathbb{R}^{n} .
$$

Then $u \notin C_{0}^{\infty}\left(\mathbb{R}^{n}\right)$ but $u \in \mathcal{S}\left(\mathbb{R}^{n}\right)$.

Remark 2.2.2. The functions in $\mathcal{S}\left(\mathbb{R}^{n}\right)$ need not have compact support however, as $x \rightarrow \infty$, all derivatives of $u$ must approach to 0 faster than any polynomial, i.e., the functions are rapidly decaying. 
Before we introduce the topological dual space of $\mathcal{S}\left(\mathbb{R}^{n}\right)$, we review some definitions. The following two definitions arise from [34].

Definition 2.2.3. A sequence $\left\{u_{j}\right\}_{j=1}^{\infty}$ of functions in the Schwartz space $\mathcal{S}\left(\mathbb{R}^{n}\right)$ is said to converge to zero in $\mathcal{S}\left(\mathbb{R}^{n}\right)$ if for all multi-indices $\alpha$ and $\beta$, we have

$$
\sup _{x \in \mathbb{R}^{n}}\left|x^{\alpha}\left(D^{\beta} u_{j}\right)(x)\right| \rightarrow 0
$$

as $j \rightarrow \infty$. We denote the convergence to zero in $\mathcal{S}\left(\mathbb{R}^{n}\right)$ by $u_{j} \rightarrow 0$ in $\mathcal{S}\left(\mathbb{R}^{n}\right)$.

Definition 2.2.4 (Tempered distribution). A linear functional $T$ on $\mathcal{S}\left(\mathbb{R}^{n}\right)$ is called a tempered distribution if for any sequence $\left\{u_{j}\right\}_{j=1}^{\infty}$ of functions in $\mathcal{S}\left(\mathbb{R}^{n}\right)$ converging to 0 in $\mathcal{S}\left(\mathbb{R}^{n}\right)$, we have

$$
T\left(u_{j}\right) \rightarrow 0
$$

as $j \rightarrow \infty$.

Consider the Schwartz space $\mathcal{S}\left(\mathbb{R}^{n}\right)$ and its topological dual space $\mathcal{S}^{\prime}\left(\mathbb{R}^{n}\right)$ consisting of tempered distributions. Consider the function $u \in \mathcal{S}\left(\mathbb{R}^{n}\right)$ and let $s$ denote a fractional parameter in $(0,1)$. Then we define the operator $(-\Delta)^{s}$ : $\mathcal{S}\left(\mathbb{R}^{n}\right) \rightarrow L^{2}\left(\mathbb{R}^{n}\right)$ of $u$ by

$$
(-\Delta)^{s} u(x):=C(n, s) \lim _{\epsilon \rightarrow 0^{+}} \int_{\mathbb{R}^{n} \backslash B(x, \epsilon)} \frac{u(x)-u(y)}{|x-y|^{n+2 s}} d y, \quad x \in \mathbb{R}^{n},
$$

where $B(x, \epsilon)$ is the ball centered at $x \in \mathbb{R}^{n}$ with radius $\epsilon$ and $C(n, s)$ denotes a normalization constant depending only on $n$ and $s$ given by

$$
C(n, s):=\left(\int_{\mathbb{R}^{n}} \frac{1-\cos \left(\zeta_{1}\right)}{|\zeta|^{n+2 s}} d \zeta\right)^{-1}, \quad \text { where } \zeta=\left(\zeta_{1}, \ldots, \zeta_{n}\right) \in \mathbb{R}^{n}
$$

We denote the fractional Laplacian by the operator $(-\Delta)^{s}$. An equivalent definition of the fractional Laplacian is given by

$$
(-\Delta)^{s} u(x):=C(n, s) \text { P.V. } \int_{\mathbb{R}^{n}} \frac{u(x)-u(y)}{|x-y|^{n+2 s}} d y, \quad x \in \mathbb{R}^{n},
$$


where P.V. denotes the Cauchy principal value and

$$
\text { P.V. } \int_{\mathbb{R}^{n}} \frac{u(x)-u(y)}{|x-y|^{n+2 s}} d y:=\lim _{\epsilon \rightarrow 0^{+}} \int_{\mathbb{R}^{n} \backslash B(x, \epsilon)} \frac{u(x)-u(y)}{|x-y|^{n+2 s}} d y .
$$

In the definition of the fractional Laplacian, we see that the integral is singular when $y$ is in the neighbourhood of $x$. For $s \in(0,1 / 2)$, the integral is not singular near $x$. We direct the interested reader to $[11,15]$ for the proof of these statements.

The singular integral can be expressed as a weighted second-order differential quotient presented in the following proposition.

Proposition 2.2.1. Let $s \in(0,1)$. Then for any $u \in \mathcal{S}\left(\mathbb{R}^{n}\right)$,

$$
(-\Delta)^{s} u(x)=-\frac{1}{2} C(n, s) \int_{\mathbb{R}^{n}} \frac{u(x+y)+u(x-y)-2 u(x)}{|y|^{n+2 s}} d y, \quad x \in \mathbb{R}^{n} .
$$

We introduce the definition of the fractional Laplacian expressed as a weighted second-order differential quotient to remove the singularity that is present in the definition of the fractional Laplacian expressed as a singular integral.

\subsubsection{The Fractional Sobolev Space $W^{s, p}$}

In this subsection, we introduce the definition of the fractional Sobolev space $W^{s, p}$ and its properties (see [11], [15]). We conclude this subsection with the fractional Sobolev space $H^{s}$ which is the special case of $W^{s, p}$ when $p=2$.

Let $\Omega$ be an open set in $\mathbb{R}^{n}$. Then for $s \in(0,1)$ and $p \in[1, \infty)$, we define the fractional Sobolev space $W^{s, p}(\Omega)$ by

$$
W^{s, p}(\Omega):=\left\{u \in L^{p}(\Omega): \frac{|u(x)-u(y)|}{|x-y|^{\frac{n}{p}+s}} \in L^{p}(\Omega \times \Omega)\right\},
$$

equipped with the norm given by

$$
\|u\|_{W^{s, p}(\Omega)}:=\left(\int_{\Omega}|u(x)|^{p} d x+\int_{\Omega} \int_{\Omega} \frac{|u(x)-u(y)|^{p}}{|x-y|^{n+s p}} d x d y\right)^{\frac{1}{p}}
$$


where the Gagliardo semi-norm of $u$ denoted by $[u]_{W^{s, p}(\Omega)}$ is defined by

$$
[u]_{W^{s, p}(\Omega)}:=\left(\int_{\Omega} \int_{\Omega} \frac{|u(x)-u(y)|^{p}}{|x-y|^{n+s p}} d x d y\right)^{\frac{1}{p}} .
$$

Recall when $s$ is expressed as an integer, we have the fact that $W^{s^{\prime}, p}$ is continuously embedded in $W^{s, p}$ for $s \leq s^{\prime}$ (see [34] Theorem 12.5). This fact also holds for $s$ expressed as a fractional parameter.

Proposition 2.2.2. Let $1 \leq p<\infty$ and $0<s \leq s^{\prime}<1$. Let $\Omega$ be an open set in $\mathbb{R}^{n}$ and $u: \Omega \rightarrow \mathbb{R}$ be a measurable function. Then

$$
\|u\|_{W^{s, p}(\Omega)} \leq C\|u\|_{W^{s^{\prime}, p}(\Omega)}
$$

for some suitable positive constant $C=C(n, s, p) \geq 1$. In particular,

$$
W^{s^{\prime}, p}(\Omega) \subseteq W^{s, p}(\Omega)
$$

(see [11] Proposition 2.1).

As with $s$ expressed as an integer, we have the fact that for $s \in(0,1)$, any function in the Sobolev space $W^{s, p}\left(\mathbb{R}^{n}\right)$ can be approximated by a sequence of smooth functions with compact support.

Theorem 2.2.5. For $s>0$, the space $C_{0}^{\infty}\left(\mathbb{R}^{n}\right)$ of smooth functions with compact support is dense in $W^{s, p}\left(\mathbb{R}^{n}\right)$. (See [11] Theorem 2.4).

In general, for $\Omega \subset \mathbb{R}^{n}, W^{s, p}(\Omega) \neq W_{0}^{s, p}(\Omega)$. In other words, $C_{0}^{\infty}(\Omega)$ is not dense in $W^{s, p}(\Omega)$. Thus we let $W_{0}^{s, p}(\Omega)$ denote the closure of $C_{0}^{\infty}(\Omega)$ with respect to the norm $\|\cdot\|_{W^{s, p}(\Omega)}$.

Similar to $s$ expressed as an integer, we can introduce the space $H^{s}(\Omega)$ where $s$ expressed as a fractional parameter. The definition of the fractional Sobolev space $H^{s}(\Omega)$ and its norm follows from (2.2) and (2.3) when $p=2$. 
Let $\Omega$ be an open set in $\mathbb{R}^{n}$. Then for $s \in(0,1)$, we define the fractional Sobolev space by

$$
H^{s}(\Omega):=\left\{u \in L^{2}(\Omega): \frac{|u(x)-u(y)|}{|x-y|^{\frac{n}{2}+s}} \in L^{2}(\Omega \times \Omega)\right\},
$$

equipped with the norm given by

$$
\|u\|_{H^{s}(\Omega)}:=\left(\int_{\Omega}|u(x)|^{2} d x+\int_{\Omega} \int_{\Omega} \frac{|u(x)-u(y)|^{2}}{|x-y|^{n+2 s}} d x d y\right)^{\frac{1}{2}},
$$

where the Gagliardo semi-norm of $u$ denoted by $[u]_{H^{s}(\Omega)}$ is defined by

$$
[u]_{H^{s}(\Omega)}:=\left(\int_{\Omega} \int_{\Omega} \frac{|u(x)-u(y)|^{2}}{|x-y|^{n+2 s}} d x d y\right)^{\frac{1}{2}} .
$$

Observe that for $p=2$, we have $W^{s, 2}(\Omega)=H^{s}(\Omega)$ which implies that $W^{s, 2}(\Omega)$ is a Hilbert space denoted by $H^{s}(\Omega)$. The inner product $(\cdot, \cdot)$ on $H^{s}(\Omega)$ is defined by

$$
(u, v)_{H^{s}(\Omega)}:=\int_{\Omega} u(x) v(x) d x+\int_{\Omega} \int_{\Omega} \frac{(u(x)-u(y))(v(x)-v(y))}{|x-y|^{n+2 s}} d x d y,
$$

for any $u, v \in H^{s}(\Omega)$. Similarly, we let $H_{0}^{s}(\Omega)$ be the closure of $C_{0}^{\infty}(\Omega)$ with respect to the norm $\|\cdot\|_{H^{s}(\Omega)}$.

\subsubsection{The Fractional Sobolev-Type Space $X^{s}$}

In this subsection, we introduce two fractional Sobolev-type spaces as well as some important properties from [15]. We shall see later that one of the Sobolevtype spaces that we will introduce in this subsection will be our space of interest when dealing with our nonlocal problem. We begin by fixing $s \in(0,1)$. Let $\Omega$ be an open bounded domain of $\mathbb{R}^{n}$ where $n>2 s$. We define the set $Q$ by

$$
Q:=\left(\mathbb{R}^{n} \times \mathbb{R}^{n}\right) \backslash\left(\left(\mathbb{R}^{n} \backslash \Omega\right) \times\left(\mathbb{R}^{n} \backslash \Omega\right)\right)
$$

In addition, let $K: \mathbb{R}^{n} \backslash\{0\} \rightarrow(0, \infty)$ be a function satisfying the following properties: 
(i) $m K \in L^{1}\left(\mathbb{R}^{n}\right)$, where $m(x):=\min \left\{|x|^{2}, 1\right\}$ and;

(ii) there exists $\theta>0$ such that $K(x) \geq \theta|x|^{-(n+2 s)}$, for any $x \in \mathbb{R}^{n} \backslash\{0\}$.

We come to our first Sobolev-type space $X^{s}(\Omega)$ which is defined by

$$
X^{s}(\Omega):=\left\{u: \mathbb{R}^{n} \rightarrow \mathbb{R}:\left.u\right|_{\Omega} \in L^{2}(\Omega),(u(x)-u(y)) \sqrt{K(x-y)} \in L^{2}(Q)\right\}
$$

where $u$ denotes Lebesgue measurable functions. The space $X^{s}(\Omega)$ is equipped with the norm given by

$$
\|u\|_{X^{s}(\Omega)}=\|u\|_{L^{2}(\Omega)}+\left(\int_{Q}|u(x)-u(y)|^{2} K(x-y) d x d y\right)^{\frac{1}{2}} .
$$

Remark 2.2.6. The norms given in (2.4) and (2.5) are not equivalent since $\Omega \times \Omega \subsetneq Q$.

The space $X_{0}^{s}(\Omega)$ is defined by

$$
X_{0}^{s}(\Omega):=\left\{u \in X^{s}(\Omega): u=0 \text { a.e. in } \mathbb{R}^{n} \backslash \Omega\right\} .
$$

Remark 2.2.7. Since we are dealing with the fractional Laplace operator $(-\Delta)^{s}$, the function $K$ is given by

$$
K(x)=|x|^{-(n+2 s)}, \quad x \in \mathbb{R}^{n} \backslash\{0\} .
$$

In addition, the Sobolev spaces $X^{s}(\Omega)$ and $X_{0}^{s}(\Omega)$ are denoted by $\mathbb{H}^{s}(\Omega)$ and $\mathbb{H}_{0}^{s}(\Omega)$ respectively.

Now, we present some properties of $X^{s}(\Omega)$ and $X_{0}^{s}(\Omega)$ without proof. For the rest of the subsection, the interested reader can refer to [15] for the proof of these properties. We begin by comparing the Sobolev space $H^{s}$ with the Sobolev spaces $X^{s}(\Omega)$ and $X_{0}^{s}(\Omega)$.

Lemma 2.2.8. Let $s \in(0,1)$ and let $K: \mathbb{R}^{n} \backslash\{0\} \rightarrow(0, \infty)$ be a function satisfying properties (i) and (ii) presented near the beginning of this subsection. Then the following assertions hold: 
(i) If $u \in X^{s}(\Omega)$, then $u \in H^{s}(\Omega)$. Moreover,

$$
\|u\|_{H^{s}(\Omega)} \leq c(\theta)\|u\|_{X^{s}(\Omega)}
$$

where $c(\theta):=\max \left\{1, \frac{1}{\sqrt{\theta}}\right\}$

(ii) If $u \in X_{0}^{s}(\Omega)$, then $u \in H^{s}\left(\mathbb{R}^{n}\right)$. Moreover,

$$
\|u\|_{H^{s}(\Omega)} \leq\|u\|_{H^{s}\left(\mathbb{R}^{n}\right)} \leq c(\theta)\|u\|_{X^{s}(\Omega)},
$$

where $c(\theta)$ is the constant presented in (i) and;

(iii) Let $K(x)=|x|^{-(n+2 s)}$. Then

$$
X_{0}^{s}(\Omega)=\left\{u \in H^{s}\left(\mathbb{R}^{n}\right): u=0 \text { a.e. in } \mathbb{R}^{n} \backslash \Omega\right\} \text {. }
$$

As with other Sobolev spaces that we have seen so far, the spaces $X^{s}(\Omega)$ and $X_{0}^{s}(\Omega)$ also satisfy the convergence property.

Lemma 2.2.9. Let $\left\{u_{j}\right\}_{j=1}^{\infty}$ be a sequence in $X^{s}(\Omega)$ such that $u_{j} \rightarrow u$ a.e. in $\mathbb{R}^{n}$ as $j \rightarrow \infty$ and

$$
\sup _{j \in \mathbb{N}}\left\|u_{j}\right\|_{X^{s}(\Omega)}<\infty .
$$

Then $u \in X^{s}(\Omega)$. In addition, if $u_{j} \in X_{0}^{s}(\Omega)$, then for any $j \in \mathbb{N}$, we have $u \in X_{0}^{s}(\Omega)$.

Finally, we shall focus on some properties for $X_{0}^{s}(\Omega)$ as this will lead up to our space of interest $\mathbb{H}_{0}^{s}(\Omega)$. Once again, we omit the proof of these properties and we direct the interested reader to [15]. Before we present some properties for $X_{0}^{s}(\Omega)$, since $u=0$ in $\mathbb{R}^{n} \backslash \Omega$, we can write the integral in (2.5) to be extended over the whole space $\mathbb{R}^{n}$. In other words, for any $u \in X_{0}^{s}(\Omega)$, we can rewrite the norm of $X^{s}(\Omega)$ as

$$
\|u\|_{X^{s}(\Omega)}=\|u\|_{L^{2}\left(\mathbb{R}^{n}\right)}+\left(\int_{\mathbb{R}^{n} \times \mathbb{R}^{n}}|u(x)-u(y)|^{2} K(x-y) d x d y\right)^{\frac{1}{2}} .
$$


Lemma 2.2.10. Let $s \in(0,1), n>2 s$, and let $K: \mathbb{R}^{n} \backslash\{0\} \rightarrow(0, \infty)$ be a function satisfying properties (i) and (ii) presented near the beginning of this subsection. Then

(i) there exists $c>0$, where $c$ depends only on $n$ and $s$, such that for any $u \in X_{0}^{s}(\Omega)$,

$$
\|u\|_{L^{2 *}(\Omega)}^{2}=\|u\|_{L^{2 *}\left(\mathbb{R}^{n}\right)}^{2} \leq c \int_{\mathbb{R}^{n} \times \mathbb{R}^{n}} \frac{|u(x)-u(y)|^{2}}{|x-y|^{n+2 s}} d x d y,
$$

where $2_{s}^{*}:=(2 n) /(n-2 s)$ is the fractional critical Sobolev exponent and;

(ii) there exists a constant $C>1$, where $C$ depends only on $n, s, \theta$, and $\Omega$ such that for any $u \in X_{0}^{s}(\Omega)$,

$$
\begin{aligned}
\int_{Q}|u(x)-u(y)|^{2} K(x-y) d x d y & \leq\|u\|_{X^{s}(\Omega)}^{2} \\
& \leq C \int_{Q}|u(x)-u(y)|^{2} K(x-y) d x d y
\end{aligned}
$$

that is

$$
\|u\|_{X_{0}^{s}(\Omega)}=\left(\int_{\mathbb{R}^{n} \times \mathbb{R}^{n}}|u(x)-u(y)|^{2} K(x-y) d x d y\right)^{\frac{1}{2}}
$$

is a norm on $X_{0}^{s}(\Omega)$ equivalent to the norm defined in (2.5).

Thus, we shall take the norm in (2.6) as a norm on $X_{0}^{s}(\Omega)$.

Lemma 2.2.11. $\left(X_{0}^{s}(\Omega),\|\cdot\|_{X_{0}^{s}(\Omega)}\right)$ is a Hilbert space with inner product defined by

$$
(u, v)_{X_{0}^{s}(\Omega)}:=\int_{\mathbb{R}^{n} \times \mathbb{R}^{n}}(u(x)-u(y))(v(x)-v(y)) K(x-y) d x d y .
$$

Since we are dealing with the fractional Laplace operator $(-\Delta)^{s}$, it follows that the norm and the inner product in $\mathbb{H}_{0}^{s}(\Omega)$ are defined by

$$
\|u\|_{\mathbb{H}_{0}^{s}(\Omega)}:=\left(\int_{\mathbb{R}^{n} \times \mathbb{R}^{n}} \frac{|u(x)-u(y)|^{2}}{|x-y|^{n+2 s}} d x d y\right)^{\frac{1}{2}}
$$


and

$$
(u, v)_{\mathbb{H}_{0}^{s}(\Omega)}:=\int_{\mathbb{R}^{n} \times \mathbb{R}^{n}} \frac{(u(x)-u(y))(v(x)-v(y))}{|x-y|^{n+2 s}} d x d y
$$

respectively. We denote the norm and inner product in $\mathbb{H}_{0}^{s}(\Omega)$ by $\|\cdot\|_{\mathbb{H}_{0}^{s}(\Omega)}$ and $(\cdot, \cdot)_{\mathbb{H}_{0}^{s}(\Omega)}$ respectively. The following lemma states a convergence property for bounded sequences in $X_{0}^{s}(\Omega)$.

Lemma 2.2.12. Let $s \in(0,1), n>2 s$, and let $\Omega$ be an open bounded subset of $\mathbb{R}^{n}$ with continuous boundary. Suppose $K: \mathbb{R}^{n} \backslash\{0\} \rightarrow(0, \infty)$ satisfy properties (i) and (ii) presented near the beginning of this subsection. In addition, let $\left\{u_{j}\right\}_{j=1}^{\infty}$ be a bounded sequence in $X_{0}^{s}(\Omega)$. Then there exists $u \in L^{q}\left(\mathbb{R}^{n}\right)$ such that, up to a subsequence,

$$
u_{j} \rightarrow u \quad \text { in } L^{q}\left(\mathbb{R}^{n}\right)
$$

as $j \rightarrow \infty$, for any $q \in\left[1,2_{s}^{*}\right)$.

\subsection{Embedding Properties}

In this section, we present the embedding properties for Lebesgue spaces, classical Sobolev spaces, fractional Sobolev spaces as well as fractional Sobolev-type spaces without proof. The importance of continuous and compact embeddings of Sobolev spaces is to aid in proving the existence of solutions of elliptic partial differential equations.

\subsubsection{The Lebesgue Space $L^{p}(\Omega)$}

We present a useful embedding theorem for $L^{p}$-spaces over domains with finite volume given in [1] without proof.

Theorem 2.3.1 (An Embedding Theorem for $L^{p}$-spaces). Suppose that

$$
\operatorname{vol}(\Omega)=\int_{\Omega} 1 d x<\infty
$$


and $1 \leq p \leq q \leq \infty$. If $u \in L^{q}(\Omega)$, then $u \in L^{p}(\Omega)$ and

$$
\|u\|_{L^{p}(\Omega)} \leq(\operatorname{vol}(\Omega))^{\left(\frac{1}{p}\right)-\left(\frac{1}{q}\right)}\|u\|_{L^{q}(\Omega)} .
$$

Hence

$$
L^{q}(\Omega) \hookrightarrow L^{p}(\Omega)
$$

If $u \in L^{\infty}(\Omega)$, then

$$
\lim _{p \rightarrow \infty}\|u\|_{L^{p}(\Omega)}=\|u\|_{L^{\infty}(\Omega)} .
$$

Finally, if $u \in L^{p}(\Omega)$ for $1 \leq p<\infty$ and if there exists a constant $C$ such that for all $p$

$$
\|u\|_{L^{p}(\Omega)} \leq C,
$$

then $u \in L^{\infty}(\Omega)$ and

$$
\|u\|_{L^{\infty}(\Omega)} \leq C .
$$

\subsubsection{The Sobolev Space $W^{k, p}(\Omega)$}

Before we state the standard embedding and compactness theorem, we briefly recall the meaning of a Banach space $V$ to be continuously and compactly embedded. Let $V$ and $X$ be Banach spaces and $V \subset X$. Then we say that $V$ is continuously embedded in $X$, written, $V \hookrightarrow X$ provided that

$$
\|u\|_{X} \leq C\|u\|_{V},
$$

for some constant $C$ and for all $u \in V$. In addition, we say that the embedding $V \hookrightarrow X$ is compact provided that

(i) $\|u\|_{X} \leq C\|u\|_{V}$, for some constant $C$ and for all $u \in V$ and;

(ii) each bounded sequence in $V$ has a converging subsequence in $X$.

Now we can state the Sobolev embedding theorem along with the standard compactness theorem known as the Rellich-Kondrachov theorem. 
Theorem 2.3.2 (The Sobolev Embedding Theorem). Let $\Omega$ be a bounded domain in $\mathbb{R}^{n}$. Let $j \geq 0$ and $m \geq 1$ be integers and let $1 \leq p<\infty$.

PART I Suppose $\Omega$ has a locally Lipschitz boundary.

Case $\boldsymbol{A}$ If either $m p>n$ or $m=n$ and $p=1$, then

$$
W^{j+m, p}(\Omega) \hookrightarrow W^{j, q}(\Omega), \quad \text { for } p \leq q \leq \infty,
$$

and, in particular,

$$
W^{m, p}(\Omega) \hookrightarrow L^{q}(\Omega), \quad \text { for } p \leq q \leq \infty .
$$

Case $\boldsymbol{B}$ If $m p=n$, then

$$
W^{j+m, p}(\Omega) \hookrightarrow W^{j, q}(\Omega), \quad \text { for } p \leq q<\infty,
$$

and, in particular,

$$
W^{m, p}(\Omega) \hookrightarrow L^{q}(\Omega), \quad \text { for } p \leq q<\infty .
$$

Case $C$ If $m p<n$, then

$$
W^{j+m, p}(\Omega) \hookrightarrow W^{j, q}(\Omega), \quad \text { for } p \leq q \leq p^{*}=\frac{n p}{n-m p} .
$$

In particular,

$$
W^{m, p}(\Omega) \hookrightarrow L^{q}(\Omega), \quad \text { for } p \leq q \leq p^{*}=\frac{n p}{n-m p} .
$$

The embedding constants for the embeddings above depend only on $n, m, p, q$, and $j$.

PART II All of the embeddings in Part I are valid for arbitrary domains $\Omega$ if the $W$-space undergoing the embedding is replaced with the corresponding $W_{0^{-}}$ space (see [1] Theorem 4.12). 
Theorem 2.3.3 (The Rellich-Kondrachov Theorem). Let $\Omega$ be a bounded domain in $\mathbb{R}^{n}$. Let $j \geq 0$ and $m \geq 1$ be integers, and let $1 \leq p<\infty$.

PART I If $\Omega$ has a locally Lipschitz boundary and $m p \leq n$, then the following embeddings are compact:

$$
\begin{aligned}
& W^{j+m, p}(\Omega) \hookrightarrow W^{j, q}(\Omega) \quad \text { if } \quad 1 \leq q<\frac{n p}{n-m p}, \\
& W^{j+m, p}(\Omega) \hookrightarrow W^{j, q}(\Omega) \quad \text { if } \quad n=m p, \quad \text { and } 1 \leq q<\infty .
\end{aligned}
$$

PART II If $\Omega$ has a locally Lipschitz boundary and $m p<n$, then the following embedding is compact:

$$
W^{j+m, p}(\Omega) \hookrightarrow W^{j, q}(\Omega) \quad \text { if } \quad 1 \leq q<\infty .
$$

PART III If $\Omega$ is an arbitrary domain in $\mathbb{R}^{n}$, the embeddings above are compact provided $W^{j+m, p}(\Omega)$ is replaced by $W_{0}^{j+m, p}(\Omega)$ (see [1] Theorem 6.3).

\subsubsection{The Fractional Sobolev Space $W^{s, p}(\Omega)$}

Before we discuss continuous and compact embeddings for fractional Sobolev spaces, we need certain regularity assumptions on the domain $\Omega$ so that we can extend any function in $W^{s, p}(\Omega)$ to a function in $W^{s, p}\left(\mathbb{R}^{n}\right)$.

Definition 2.3.4 (Extension domain). For any $s \in(0,1)$ and $p \in[1, \infty)$, we say that an open set $\Omega \subset \mathbb{R}^{n}$ is an extension domain for $W^{s, p}$ if there exists a positive constant $C=C(n, p, s, \Omega)$ such that for every function $u \in W^{s, p}(\Omega)$, there exists $\mathcal{E}_{u} \in W^{s, p}\left(\mathbb{R}^{n}\right)$ such that $\mathcal{E}_{u}(x)=u(x)$ for any $x \in \Omega$ and

$$
\left\|\mathcal{E}_{u}\right\|_{W^{s, p}\left(\mathbb{R}^{n}\right)} \leq C\|u\|_{W^{s, p}(\Omega)}
$$

(see [15] Definition 1.2).

The following lemma states the construction of the extension $\mathcal{E}_{u}$ to the whole space of $\mathbb{R}^{n}$. 
Lemma 2.3.5. Let $\Omega$ be an open set in $\mathbb{R}^{n}$, and let $u \in W^{s, p}(\Omega)$ with $s \in(0,1)$ and $p \in[1, \infty)$. If there exists a compact subset $\mathcal{K} \subset \Omega$ such that $u \equiv 0$ in $\Omega \backslash \mathcal{K}$, then the extension function $\mathcal{E}_{u}$ defined as

$$
\mathcal{E}_{u}(x):= \begin{cases}u(x) & \text { if } x \in \Omega, \\ 0 & \text { if } x \in \mathbb{R}^{n} \backslash \Omega .\end{cases}
$$

belongs to $W^{s, p}\left(\mathbb{R}^{n}\right)$, and

$$
\left\|\mathcal{E}_{u}\right\|_{W^{s, p}\left(\mathbb{R}^{n}\right)} \leq C\|u\|_{W^{s, p}(\Omega)}
$$

where $C$ is a suitable positive constant depending on $n, p, s, \mathcal{K}$, and $\Omega$ (see [15] Lemma 1.3).

Now we present a theorem omitting its proof which states that every open bounded set $\Omega$ with Lipschitz boundary is an extension domain for $W^{s, p}$. Once again, the interested reader can refer to [11] for the proof of the theorem.

Theorem 2.3.6. Let $p \in[1, \infty), s \in(0,1)$ and $\Omega \subset \mathbb{R}^{n}$ be an open set of class $C^{0,1}$ with bounded boundary. Then $W^{s, p}(\Omega)$ is continuously embedded in $W^{s, p}\left(\mathbb{R}^{n}\right)$, namely for any $u \in W^{s, p}(\Omega)$ there exists $\mathcal{E}_{u} \in W^{s, p}\left(\mathbb{R}^{n}\right)$ such that $\left.\mathcal{E}_{u}\right|_{\Omega}=u$ and

$$
\left\|\mathcal{E}_{u}\right\|_{W^{s, p}\left(\mathbb{R}^{n}\right)} \leq C\|u\|_{W^{s, p}(\Omega)}
$$

where $C=C(n, p, s, \Omega)$.

We can now introduce continuous and compact embeddings for fractional Sobolev spaces presented in two cases; the first being the case when $s p<n$ and the second being the case when $s p=n$. We only focus on the space $W^{s, p}(\Omega)$ where $\Omega$ is an extension domain of $\mathbb{R}^{n}$. We direct the interested reader to $[11,15]$ for the space $W^{s, p}\left(\mathbb{R}^{n}\right)$. The following theorem deals with the case when $s p<n$. Theorem 2.3.7. Let $s \in(0,1)$ and $p \in[1, \infty)$ such that $s p<n$. Let $\Omega \subset \mathbb{R}^{n}$ be an extension domain for $W^{s, p}$. Then there exists a positive constant $C:=$ 
$C(n, p, s, \Omega)$ such that, for any $u \in W^{s, p}(\Omega)$,

$$
\|u\|_{L^{q}(\Omega)} \leq C\|u\|_{W^{s, p}(\Omega)}
$$

for any $q \in\left[p, p_{s}^{*}\right]$ where the constant $p_{s}^{*}:=(n p) /(n-s p)$ is the fractional critical Sobolev exponent; that is, the space $W^{s, p}(\Omega)$ is continuously embedded in $L^{q}(\Omega)$ for any $q \in\left[p, p_{s}^{*}\right]$. If in addition, $\Omega$ is bounded, then the space $W^{s, p}(\Omega)$ is compactly embedded in $L^{q}(\Omega)$ for any $q \in\left[1, p_{s}^{*}\right)$ (see [15] Theorem 1.5).

For the case when $s p=n$, we have the following continuous and compact embedding theorem.

Theorem 2.3.8. Let $s \in(0,1)$ and $p \in[1, \infty)$ be such that $s p=n$. Let $\Omega \subset \mathbb{R}^{n}$ be an extension domain for $W^{s, p}$. Then there exists a positive constant $C:=$ $C(n, p, s, \Omega)$ such that, for any $u \in W^{s, p}(\Omega)$,

$$
\|u\|_{L^{q}(\Omega)} \leq C\|u\|_{W^{s, p}(\Omega)},
$$

for any $q \in[p, \infty)$; that is, the space $W^{s, p}(\Omega)$ is continuously embedded in $L^{q}(\Omega)$ for any $q \in[p, \infty)$. If, in addition, $\Omega$ is bounded, then the space $W^{s, p}(\Omega)$ is compactly embedded in $L^{q}(\Omega)$ for any $q \in[1, \infty)$ (see [15] Theorem 1.7).

\subsubsection{The Fractional Sobolev-type Space $X_{0}^{s}(\Omega)$}

Finally, we present an embedding property for $X_{0}^{s}(\Omega)$ into the classical Lebesgue spaces. The following lemma summarizes the results presented in Lemma 2.2.10(i) and Lemma 2.2.12.

Lemma 2.3.9. Let $s \in(0,1), n>2 s$, and let $K: \mathbb{R}^{n} \backslash\{0\} \rightarrow(0, \infty)$ be a function satisfying the properties presented near the beginning of Subsection 2.2.3. Then the following assertions hold:

(i) If $\Omega$ has a continuous boundary, then the embedding $X_{0}^{s}(\Omega) \hookrightarrow L^{q}(\Omega)$ is compact for any $q \in\left[1,2_{s}^{*}\right)$ and; 
(ii) The embedding $X_{0}^{s}(\Omega) \hookrightarrow L^{2_{s}^{*}}(\Omega)$ is continuous

(see [15] Lemma 1.31).

\subsection{Regularity Theory}

Finally, after proving the existence of a weak solution of partial differential equations, we need to address whether this weak solution is in fact smooth. In order to see this, we use certain regularity results to address this problem. We begin this section with some definitions on second-order elliptic equations from [13], leading up to the definition of a weak solution also found in [13]. We then take a look at an example of a semilinear elliptic equation along with the definition of a weak solution arising from [33]. We end this section with two regularity results presented in $[6,14]$.

Consider the following boundary value problem

$$
\begin{cases}L u=f & \text { in } \Omega \\ u=0, & \text { on } \partial \Omega\end{cases}
$$

where $\Omega$ is an open bounded subset of $\mathbb{R}^{n}, u: \bar{\Omega} \rightarrow \mathbb{R}$ is the unknown, and $f: \Omega \rightarrow \mathbb{R}$ is given. Let $L$ denote a second-order partial differential operator possessing either divergence or nondivergence form given by

$$
L u=-\sum_{i, j=1}^{n}\left(a^{i j}(x) u_{x_{j}}+b^{i}(x) u\right)_{x_{i}}+\sum_{i=1}^{n} c^{i}(x) u_{x_{i}}+d(x) u
$$

and

$$
L u=-\sum_{i, j=1}^{n} a^{i j}(x) u_{x_{i} x_{j}}+\sum_{i=1}^{n} b^{i}(x) u_{x_{i}}+c(x) u
$$

respectively, where $a^{i j}, b^{i}, c^{i}, d$ are given coefficient functions and $i, j=1, \ldots, n$. In addition, we assume the symmetry condition

$$
a^{i j}=a^{j i}, \quad \text { for } i, j=1, \ldots, n \text {. }
$$


Definition 2.4.1. We say the partial differential operator $L$ is strictly elliptic if there exists a constant $\theta>0$ such that

$$
\sum_{i, j=1}^{n} a^{i j}(x) \xi_{i} \xi_{j} \geq \theta|\xi|^{2}
$$

for a.e. $x \in \Omega$ and all $\xi \in \mathbb{R}^{n}$.

In other words, we say that $L$ is elliptic at a point $x \in \Omega$ if the symmetric matrix $A(x)=\left[a^{i j}(x)\right]$ is positive definite with smallest eigenvalue greater than or equal to $\theta$. Before we provide a definition of a weak solution to (2.7), we need to define the bilinear form denoted by $B[\cdot, \cdot]$.

Definition 2.4.2 (Bilinear form). Consider the boundary-value problem (2.7) when $L$ has the divergence form (2.8). Suppose $a^{i j}, b^{i}, c^{i}, d \in L^{\infty}(\Omega)$ where $i, j=$ $1, \ldots, n$ and $f \in L^{2}(\Omega)$. Then the bilinear form $B[\cdot, \cdot]$ associated with the divergence form elliptic operator $L$ defined by (2.8) is

$$
B[u, v]:=\int_{\Omega} \sum_{i, j=1}^{n}\left(a^{i j}(x) u_{x_{j}}+b^{i}(x) u\right) v_{x_{i}}+\sum_{i=1}^{n} c^{i}(x) u_{x_{i}} v+d(x) u v d x
$$

for $u, v \in H_{0}^{1}(\Omega)$.

Now we can give a formal definition of weak solutions.

Definition 2.4.3 (Weak solution). Consider the boundary-value problem (2.7) and the assumptions stated in Definition 2.4.2. We say that $u \in H_{0}^{1}(\Omega)$ is a weak solution of the boundary-value problem (2.7) if

$$
B[u, v]=(f, v)
$$

for all $u, v \in H_{0}^{1}(\Omega)$, where $(\cdot, \cdot)$ denotes the inner product in $L^{2}(\Omega)$.

Now, let us consider a more general boundary-value problem

$$
\begin{cases}L u=f^{0}-\sum_{i=1}^{n} f_{x_{i}}^{i} & \text { in } \Omega \\ u=0, & \text { on } \partial \Omega .\end{cases}
$$


where $L$ has the divergence form $(2.8)$ and $f^{i} \in L^{2}(\Omega)$ for $i=1, \ldots, n$. We define a weak solution associated to problem (2.10) which we find useful in our study of the existence of a weak solution for our local problem.

Definition 2.4.4 (Weak solution). We say $u \in H_{0}^{1}(\Omega)$ is a weak solution of problem (2.10) provided

$$
B[u, v]=\langle f, v\rangle
$$

for all $v \in H_{0}^{1}(\Omega)$, where

$$
\langle f, v\rangle=\int_{\Omega} f^{0} v+\sum_{i=1}^{n} f^{i} v_{x_{i}} d x
$$

and $\langle\cdot, \cdot\rangle$ is the pairing of $H_{0}^{1}(\Omega)$ and its topological dual space $H^{-1}(\Omega)$.

Finally, we consider the following semilinear elliptic problem involving the fractional Laplacian

$$
\begin{cases}(-\Delta)^{s} u=f(x, u), & x \in \Omega, \\ u=0, & x \in \mathbb{R}^{n} \backslash \Omega .\end{cases}
$$

where $\Omega \subset \mathbb{R}^{n}, n \geq 2$ is an open bounded domain with $C^{2}$-boundary, $s \in(0,1)$, and $(-\Delta)^{s}$ denotes the fractional Laplacian. We define a weak solution associated to problem (2.11) which we find useful in our study of the existence of a weak solution for our nonlocal problem.

Definition 2.4.5 (Weak solution). We say $u \in \mathbb{H}_{0}^{s}(\Omega)$ is a weak solution of problem (2.11) if u satisfies

$$
\int_{\mathbb{R}^{n} \times \mathbb{R}^{n}} \frac{(u(x)-u(y))(v(x)-v(y))}{|x-y|^{n+2 s}} d x d y=\int_{\Omega} f(x, u(x)) v(x) d x,
$$

for all $v \in \mathbb{H}_{0}^{s}(\Omega)$.

A weak solution $u$ is equivalent to being a critical point of the functional

$$
I[u]=\frac{1}{2} \int_{Q} \frac{|u(x)-u(y)|^{2}}{|x-y|^{n+2 s}} d x d y-\int_{\Omega} F(x, u(x)) d x,
$$


where $F(x, u)=\int_{0}^{u} f(x, s) d s$. Indeed, for any $v \in \mathbb{H}_{0}^{s}(\Omega)$, we get

$$
\left(I^{\prime}[u], v\right)=\int_{\mathbb{R}^{n} \times \mathbb{R}^{n}} \frac{(u(x)-u(y))(v(x)-v(y))}{|x-y|^{n+2 s}} d x d y-\int_{\Omega} f(x, u(x)) v(x) d x .
$$

Thus, critical points of the functional $I$ are weak solutions to problem (2.11).

We end this section by presenting the regularity results needed for the proof of our local and nonlocal problems. We begin with the first regularity result which is the elliptic regularity theorem provided in [14].

Theorem 2.4.6 (Elliptic regularity). Let $u \in W^{1,2}(\Omega)$ be a weak solution of the equation $L u=f$ in $\Omega$ where $L$ is strictly elliptic in $\Omega$, the coefficients $a^{i j}, b^{i}, i, j=$ $1, \ldots, n$ are uniformly Lipschitz continuous in $\Omega$, the coefficients $c^{i}, d, i=1, \ldots, n$ are essentially bounded in $\Omega$ and the function $f$ is in $L^{2}(\Omega)$. In addition, let us assume that $\partial \Omega$ is of class $C^{2}$ and that there exists a function $\phi \in W^{2,2}(\Omega)$ for which $u-\phi \in W_{0}^{1,2}(\Omega)$. Then we have also $u \in W^{2,2}(\Omega)$ and

$$
\|u\|_{W^{2,2}(\Omega)} \leq C\left(\|u\|_{L^{2}(\Omega)}+\|f\|_{L^{2}(\Omega)}+\|\phi\|_{W^{2,2}(\Omega)}\right)
$$

where $C=C(n, \theta, K, \partial \Omega)$, and $K$ are given by

$$
K:=\max \left\{\left\|a^{i j}, b^{i}\right\|_{C^{0,1}(\bar{\Omega})},\left\|c^{i}, d\right\|_{L^{\infty}(\Omega)}\right\}
$$

(see [14] Theorem 8.12).

For problems involving nonlocal operators, that is, in our case the fractional Laplacian $(-\Delta)^{s}$, we present the $L^{q}$-regularity of weak solutions provided in [6].

Lemma 2.4.7. Suppose that $n>2 s$ and $f \in L^{t}(\Omega)$ for some $t \geq \frac{2 n}{n+2 s}$. Then the following Dirichlet problem

$$
\begin{cases}(-\Delta)^{s} u=f, & \text { in } \Omega, \\ u=0, & \text { on } \mathbb{R}^{n} \backslash \Omega\end{cases}
$$

has a unique weak solution $u$. In addition, the following assertions hold: 
(i) If $t>\frac{n}{2 s}$, then $u \in L^{\infty}(\Omega)$ and there exists a constant $C>0$ such that

$$
\|u\|_{L^{\infty}(\Omega)} \leq C\|f\|_{L^{t}(\Omega)}
$$

(ii) If $\frac{2 n}{n+2 s} \leq t \leq \frac{n}{2 s}$, then $u \in L^{q}(\Omega)$ for every $q$ satisfying $t \leq q<\frac{n t}{n-2 s t}$ and there exists a constant $C>0$ such that

$$
\|u\|_{L^{q}(\Omega)} \leq C\|f\|_{L^{t}(\Omega)}
$$

(see [6] Lemma 2.5).

\subsection{Convex Analysis}

As we shall see later in Chapter 4, we need some important definitions and results arising from convex analysis for lower semi-continuous functions.

Let $V$ be a real Banach space, $V^{*}$ be its topological dual, and let $\langle.,$.$\rangle denote$ the pairing of $V$ and $V^{*}$. Let the weak topology on $V$ induced by $\langle.,$.$\rangle be denoted$ by $\sigma\left(V, V^{*}\right)$.

Definition 2.5.1 (Convex set). A subset $K$ of $\mathbb{R}^{n}$ is said to be convex if

$$
(1-\lambda) u+\lambda v \in K
$$

whenever $u \in K, v \in K$ and $0<\lambda<1$ (see [25] Pg. 10).

Definition 2.5.2 (Convex function). A function $u: \mathbb{R}^{n} \rightarrow \mathbb{R}$ is called convex if for all $x, y \in \mathbb{R}^{n}$ and for each $0 \leq \lambda \leq 1$

$$
u(\lambda x+(1-\lambda) y) \leq \lambda u(x)+(1-\lambda) u(y)
$$

(see [13] on Pg. 621).

We say that a convex function $u$ is proper if it nowhere takes the value $-\infty$ and is not identically $\infty$. We refer the interested reader to [12] for further details. 
Definition 2.5.3 (Coercive). A function $F$ is called coercive over a nonempty closed convex subset $K$ of $V$ if

$$
\lim _{\|u\| \rightarrow \infty} F(u)=\infty \quad \text { for } u \in K
$$

(see [12]) Proposition 1.1).

Definition 2.5.4. A function $F: V \rightarrow \mathbb{R}$ is said to be weakly lower semicontinuous if for each $u \in V$ and any sequence $\left\{u_{j}\right\}_{j=1}^{\infty}$ converging to $u$ in the weak topology $\sigma\left(V, V^{*}\right)$,

$$
F(u) \leq \liminf _{j \rightarrow \infty} F\left(u_{j}\right)
$$

Before we define the subdifferential of $F$ at $u$, let $F: V \rightarrow(-\infty, \infty]$ be a convex and weakly lower semi-continuous function. We define the effective domain of $F$ by

$$
\operatorname{Dom}(F)=\{u \in V: F(u)<\infty\}
$$

Definition 2.5.5. Let $F: V \rightarrow(-\infty, \infty]$ be a proper convex function. The subdifferential $\partial F$ of $F$ is defined to be the following set-valued operator: if $u \in$ $\operatorname{Dom}(F)=\{v \in V ; F(v)<\infty\}$, set

$$
\partial F(u)=\left\{u^{*} \in V^{*}: F(v)-F(u) \geq\left\langle u^{*}, v-u\right\rangle \text { for all } v \in V\right\}
$$

and if $u \notin \operatorname{Dom}(F)$, set $\partial F(u)=\emptyset$. If $F$ is Gâteaux differentiable at $u$, we denote the derivative of $F$ at $u$ by $D F(u)$. In this case $\partial F(u)=\{D F(u)\}$ (see [31] Pg. 78).

Now we define Gâteaux differentiability of a function $F$ at $u$ as follows.

Definition 2.5.6 (Gâteaux Differentiability). Let $F$ be a function of $V$ into $\mathbb{R} \cup\{\infty\}$. We call the limit as $\lambda \rightarrow 0_{+}$, if it exists, of

$$
\frac{F(u+\lambda v)-F(u)}{\lambda}
$$


the directional derivative of $F$ at $u$ in the direction $v$ and denote it by $F^{\prime}(u ; v)$. If there exists $u^{*} \in V^{*}$ such that:

$$
\forall v \in V, \quad F^{\prime}(u ; v)=\left\langle v, u^{*}\right\rangle
$$

we say that $F$ is Gâteaux differentiable at $u$, call $u^{*}$ the Gâteaux differential at $u$ of $F$, and denote it by $F^{\prime}(u)$ (see [12] Definition 5.2).

Definition 2.5.7. Let $V$ be a real Banach space, $\Phi \in C^{1}(V, \mathbb{R})$ and $\Psi: V \rightarrow$ $(-\infty,+\infty]$ be proper (i.e. Dom $(\Psi) \neq \emptyset)$, convex and lower semi-continuous. $A$ point $u \in V$ is said to be a critical point of

$$
I:=\Psi-\Phi
$$

if $u \in \operatorname{Dom}(\Psi)$ and if it satisfies the inequality

$$
\Psi(v)-\Psi(u)-\langle D \Phi(u), v-u\rangle \geq 0, \quad \forall v \in V
$$

(see [31] on $\mathrm{Pg}$. 80).

After the extensive concepts presented in this chapter, we can now begin with the main purpose of the thesis. The next chapter introduces the new variational principle arising from [17]. 


\section{Chapter 3}

\section{A New Variational Principle}

Let $V$ be a Banach space and $V^{*}$ be its topological dual space. Let $A: V \rightarrow V^{*}$ be possibly a nonlinear mapping from $V$ onto $V^{*}$. Given a particular class of functional equations presented in the abstract form

$$
A[u]=0,
$$

where $u$ is the unknown, we call such equations to be of variational form if we can rewrite $A[\cdot]$ in (3.1) to be the derivative of a suitable energy functional $I: V \rightarrow \mathbb{R}$ denoted by $I^{\prime}[\cdot]$. Thus, (3.1) turns out to be

$$
I^{\prime}[u]=0 .
$$

Looking at (3.2), this simply means that solutions of (3.1) are just critical points of $I[\cdot]$.

Let $V$ be a real Banach space, $V^{*}$ be its topological dual, and $\langle.,$.$\rangle denote$ the pairing of $V$ and $V^{*}$. Let $K$ be a convex and weakly closed subset of $V$. Let $\Psi: V \rightarrow(-\infty, \infty]$ be a proper convex and lower semi-continuous function which is Gâteaux differentiable on $K$ denoted by $D \Psi$. The restriction of $\Psi$ to $K$ is denoted by $\Psi_{K}$ and is defined by

$$
\Psi_{K}(u)= \begin{cases}\Psi(u), & u \in K, \\ +\infty, & u \notin K .\end{cases}
$$


Let $\Phi \in C^{1}(V, \mathbb{R})$ and consider the functional $I_{K}: V \rightarrow(-\infty, \infty]$ defined by

$$
I_{K}[u]:=\Psi_{K}(u)-\Phi(u) .
$$

The following definition for critical points of $I_{K}$ is given by Szulkin [31].

Definition 3.0.1. A point $u \in V$ is said to be a critical point of $I_{K}$ if $I_{K}[u] \in \mathbb{R}$ and if it satisfies the inequality

$$
\Psi_{K}(v)-\Psi_{K}(u) \geq\langle D \Phi(u), v-u\rangle, \quad \forall v \in V .
$$

Before we state and prove the new variational principle, we need the following proposition by Ekeland and Temam without proof.

Proposition 3.0.1. Let $\Psi: V \rightarrow(-\infty, \infty]$ be convex and lower semi-continuous. Then the following holds:

$$
\Psi(u)+\Psi^{*}\left(u^{*}\right)=\left\langle u, u^{*}\right\rangle \Longleftrightarrow u^{*} \in \partial \Psi(u)
$$

(see [12] Proposition 5.1).

The notation $\Psi^{*}$ represents the Fenchel dual of an arbitrary function $\Psi$ such that $\Psi^{*}: V^{*} \rightarrow(-\infty, \infty]$ is defined by

$$
\Psi^{*}\left(u^{*}\right)=\sup _{u \in V}\left\{\left\langle u^{*}, u\right\rangle-\Psi(u)\right\} .
$$

We introduce in this section a new variational principle and its proof provided in [17] which allows one to deal with problems well beyond the weakly compact structure.

The following theorem is the new variational principle introduced in [17].

Theorem 3.0.2 (Variational Principle). Let $V$ be a real Banach space and $K$ be a convex and weakly closed subset of $V$. Let $\Psi: V \rightarrow(-\infty, \infty]$ be a proper convex and lower semi-continuous function which is Gatteaux differentiable on $K$ and $\Phi \in C^{1}(V, \mathbb{R})$. Suppose the following two assertions hold: 
(i) The functional $I_{K}: V \rightarrow(-\infty, \infty]$ defined by $I_{K}[u]=\Psi_{K}(u)-\Phi(u)$ has a critical point $u_{0} \in V$, and;

(ii) there exists $v_{0} \in K$ such that $D \Psi\left(v_{0}\right)=D \Phi\left(u_{0}\right)$.

Then $u_{0} \in K$ is a solution of the equation

$$
D \Psi(u)=D \Phi(u)
$$

Proof. Since $u_{0}$ is a critical point of $I[u]=\Psi_{K}(u)-\Phi(u)$, it follows from Definition 2.5 .7

$$
\Psi_{K}(v)-\Psi_{K}\left(u_{0}\right) \geq\left\langle D \Phi\left(u_{0}\right), v-u_{0}\right\rangle, \quad \forall v \in V
$$

By Theorem 3.0.2 (i) and (ii), $u_{0}, v_{0} \in K$ and $D \Psi\left(v_{0}\right)=D \Phi\left(u_{0}\right)$. Setting $v=v_{0}$, it follows from (3.3) that

$$
\Psi\left(v_{0}\right)-\Psi\left(u_{0}\right) \geq\left\langle D \Psi\left(v_{0}\right), v_{0}-u_{0}\right\rangle
$$

Recall that $\Psi$ is Gâteaux differentiable at $v_{0} \in K$. Thus, it follows from Definition 2.5.5 together with the convexity of $\Psi$ that we obtain

$$
\Psi\left(u_{0}\right)-\Psi\left(v_{0}\right) \geq\left\langle D \Psi\left(v_{0}\right), u_{0}-v_{0}\right\rangle
$$

. By (3.4) and (3.5), we get

$$
\Psi\left(v_{0}\right)-\Psi\left(u_{0}\right)=\left\langle D \Psi\left(v_{0}\right), v_{0}-u_{0}\right\rangle
$$

Now, we claim that $D \Psi\left(v_{0}\right)=D \Psi\left(u_{0}\right)$, from which the desired result follows,

$$
D \Psi\left(u_{0}\right)=D \Psi\left(v_{0}\right)=D \Phi\left(u_{0}\right)
$$

To see that the claim holds, let $w^{*}=D \Psi\left(v_{0}\right)$. Since $\Psi$ is convex and lower semi-continuous, it follows from Proposition 3.0.1 that

$$
\Psi\left(v_{0}\right)+\Psi^{*}\left(w^{*}\right)=\left\langle w^{*}, v_{0}\right\rangle
$$


By (3.6) and (3.7), we get

$$
\left\langle w^{*}, u_{0}\right\rangle-\Psi\left(u_{0}\right)=\left\langle w^{*}, v_{0}\right\rangle-\Psi\left(v_{0}\right)=\Psi^{*}\left(w^{*}\right)
$$

from which we obtain

$$
\Psi\left(u_{0}\right)+\Psi^{*}\left(w^{*}\right)=\left\langle w^{*}, u_{0}\right\rangle
$$

Indeed, this implies that $w^{*} \in \partial \Psi\left(u_{0}\right)$ by Proposition 3.0.1. Since $\Psi$ is Gâteaux differentiable at $u_{0}$, it follows that $\partial \Psi\left(u_{0}\right)=\left\{D \Psi\left(u_{0}\right)\right\}$. Thus,

$$
D \Psi\left(u_{0}\right)=w^{*}=D \Psi\left(v_{0}\right),
$$

which proves our claim. 


\section{Chapter 4}

\section{Nonhomogeneous Local and Nonlocal Semilinear Elliptic \\ Equations}

In this chapter, we study the existence of a solution for the following semilinear elliptic problem given by

$$
\begin{cases}-\Delta u=|u|^{p-2} u+f(x), & x \in \Omega, \\ u=0, & x \in \partial \Omega,\end{cases}
$$

where $\Omega$ is an open bounded domain in $\mathbb{R}^{n}$ with $C^{2}$-boundary and $f \in L^{2}(\Omega)$.

In addition to the problem presented in (4.1), we are also interested in the existence of a solution for the following semilinear elliptic problem

$$
\begin{cases}(-\Delta)^{s} u=|u|^{p-2} u+f(x), & x \in \Omega, \\ u=0, & x \in \mathbb{R}^{n} \backslash \Omega,\end{cases}
$$

where $(-\Delta)^{s}$ denotes the fractional Laplace operator with $s \in(0,1), n>2 s, \Omega$ is an open bounded domain in $\mathbb{R}^{n}$ with $C^{2}$-boundary and $f \in L^{2}(\Omega)$. In both cases, we shall deal with supercritical values of $p$ by means of Sobolev spaces. 


\subsection{Semilinear Elliptic Equation Involving the Classical Laplacian}

In this section, we prove the existence of a weak solution for the following nonhomogeneous semilinear elliptic problem

$$
\begin{cases}-\Delta u=|u|^{p-2} u+f(x), & x \in \Omega, \\ u=0, & x \in \partial \Omega\end{cases}
$$

via a new variational principle in [17]. In order to utilize Theorem 3.0.2, we need to set-up our problem.

Consider the Banach space $V=H^{2}(\Omega) \cap H_{0}^{1}(\Omega)$ equipped with the norm,

$$
\|u\|_{V}:=\|u\|_{H^{2}(\Omega)}+\|u\|_{H_{0}^{1}(\Omega)}
$$

and consider $V^{*}$ to be the topological dual of $V$. Let $I: V \rightarrow \mathbb{R}$ be the EulerLagrange functional corresponding to (4.3),

$$
I[u]=\frac{1}{2} \int_{\Omega}|\nabla u|^{2} d x-\frac{1}{p} \int_{\Omega}|u|^{p} d x-\int_{\Omega} f u d x .
$$

Working with this specific Banach space, we consider the convex and weakly closed subset $K$ of $V$ defined by

$$
K:=K(r)=\left\{u \in V:\|u\|_{H^{2}(\Omega)} \leq r\right\}
$$

for some $r>0$ to be determined. To verify that $K$ is convex and weakly closed, we present the proof of the statement in two lemmas.

Lemma 4.1.1. The set $K$ defined by

$$
K:=K(r)=\left\{u \in V:\|u\|_{H^{2}(\Omega)} \leq r\right\}
$$

for some $r>0$ to be determined, is convex. 
Proof. Let $K:=K(r)=\left\{u \in V:\|u\|_{H^{2}(\Omega)} \leq r\right\}$. Let $u_{1}, u_{2} \in K$. Then

$$
\left\{\begin{array}{l}
\left\|u_{1}\right\|_{H^{2}(\Omega)} \leq r \\
\left\|u_{2}\right\|_{H^{2}(\Omega)} \leq r
\end{array}\right.
$$

for some $r>0$ to be determined. Let $0<\lambda<1$. Then

$$
\begin{aligned}
\left\|(1-\lambda) u_{1}+\lambda u_{2}\right\|_{H^{2}(\Omega)} & \leq\left\|(1-\lambda) u_{1}\right\|_{H^{2}(\Omega)}+\left\|\lambda u_{2}\right\|_{H^{2}(\Omega)} \\
& =|1-\lambda|\left\|u_{1}\right\|_{H^{2}(\Omega)}+|\lambda|\left\|u_{2}\right\|_{H^{2}(\Omega)} .
\end{aligned}
$$

Since $\left\|u_{1}\right\|_{H^{2}(\Omega)} \leq r$ and $\left\|u_{2}\right\|_{H^{2}(\Omega)} \leq r$, it follows that

$$
\left\|(1-\lambda) u_{1}+\lambda u_{2}\right\|_{H^{2}(\Omega)} \leq(1-\lambda) r+\lambda r=r .
$$

Thus, $K:=K(r)=\left\{u \in V:\|u\|_{H^{2}(\Omega)} \leq r\right\}$ is convex.

Lemma 4.1.2. The set $K$ defined in Lemma 4.1.1 is weakly closed.

Proof. Let $\left\{u_{j}\right\}_{j=1}^{\infty}$ be a sequence in $K(r)$ such that

$$
u_{j} \rightarrow u \quad \text { weakly in } V .
$$

It follows that, up to a subsequence of $u_{j}$, call it $u_{j}$, we have

$$
u_{j} \rightarrow u \quad \text { a.e. in } \Omega \text {. }
$$

But $\left\{u_{j}\right\}_{j=1}^{\infty} \subset K(r)$ implies that $\left\|u_{j}\right\|_{H^{2}(\Omega)} \leq r$. So we can conclude that $\left\{u_{j}\right\}_{j=1}^{\infty}$ is bounded in $H^{2}(\Omega)$. Once again up to a subsequence, there exists $u_{0} \in H^{2}(\Omega)$ such that

$$
u_{j} \rightarrow u_{0} \quad \text { weakly in } H^{2}(\Omega)
$$

and

$$
u_{j} \rightarrow u_{0} \quad \text { a.e. in } \Omega .
$$

So we get $u=u_{0}$ a.e. in $\Omega$ and thus $u_{j} \rightarrow u$ weakly in $H^{2}(\Omega)$. By the weak lower semi-continuity of the norm in $H^{2}(\Omega)$, we obtain

$$
\|u\|_{H^{2}(\Omega)} \leq \liminf _{j \rightarrow \infty}\left\|u_{j}\right\|_{H^{2}(\Omega)} \leq r .
$$

Therefore, $u \in K(r)$ and so $K(r)$ is weakly closed. 
Redirecting back to the set-up of the problem, we define $\Phi: V \rightarrow \mathbb{R}$ by

$$
\Phi(u)=\frac{1}{p} \int_{\Omega}|u|^{p} d x+\int_{\Omega} f u d x,
$$

where $\Phi \in C^{1}(V, \mathbb{R})$ and $\Psi: V \rightarrow \mathbb{R}$ by

$$
\Psi(u)=\frac{1}{2} \int_{\Omega}|\nabla u|^{2} d x,
$$

where $\Psi$ is a proper convex and lower semi-continuous function which is Gâteaux differentiable on $K$. We define the restriction of $\Psi$ to $K$ by

$$
\Psi_{K}(u)=\left\{\begin{array}{cc}
\frac{1}{2} \int_{\Omega}|\nabla u|^{2} d x, & u \in K, \\
+\infty, & u \notin K,
\end{array}\right.
$$

and denote the restriction of $\Psi$ to $K$ by $\Psi_{K}$.

Finally, we shall consider the critical points of the functional $I_{K}: V \rightarrow$ $(-\infty, \infty]$ where $I_{K}$ is defined by

$$
I_{K}[u]:=\Psi_{K}(u)-\Phi(u) .
$$

Now we can apply the variational principle specific to our problem.

Corollary 4.1.3. Let $V=H^{2}(\Omega) \cap H_{0}^{1}(\Omega)$ and $K$ be a convex and weakly closed subset of V. Let $\Phi$ and $\Psi$ be given by (4.4) and (4.5) respectively. Suppose the following two assertions hold:

(i) The functional $I_{K}: V \rightarrow(-\infty, \infty]$ defined by (4.7) has a critical point $u_{0} \in V$, and;

(ii) there exists $v_{0} \in K$ such that $-\Delta v_{0}=\left|u_{0}\right|^{p-2} u_{0}+f(x)$.

Then $u_{0} \in K$ is a solution of the equation

$$
-\Delta u=|u|^{p-2} u+f(x) .
$$

Before we prove Corollary 4.1.3, we need a proposition by Szulkin ([31]) which states that if $u$ is a relative minimum of $I$, then $u$ is a critical point of $I$. 
Proposition 4.1.1. If I satisfies the following hypothesis:

$I=\Psi-\Phi$, where $\Phi \in C^{1}(V, \mathbb{R})$ and $\Psi: V \rightarrow(-\infty, \infty]$ is a proper convex and lower semi-continuous function.

Then each relative minimum is necessarily a critical point of I.

Proof. Let $u$ be a relative minimum of $I$. Since $\Psi$ is convex, it follows that for all small $t>0$ and $v \in V$,

$$
\begin{aligned}
0 & \leq I[(1-t) u+t v]-I[u] \\
& =\Psi((1-t) u+t v)-\Phi((1-t) u+t v)-\Psi(u)+\Phi(u) \\
& \leq(1-t) \Psi(u)+t \Psi(v)-\Phi((1-t) u+t v)-\Psi(u)+\Phi(u) \\
& =t(\Psi(v)-\Psi(u))-\Phi((1-t) u+t v)+\Phi(u) .
\end{aligned}
$$

Dividing by $t$ we get,

$$
\Psi(v)-\Psi(u)-\frac{\Phi((1-t) u+t v)+\Phi(u)}{t} \geq 0 .
$$

Letting $t \rightarrow 0^{+}$we get,

$$
\Psi(v)-\Psi(u)-\lim _{t \rightarrow 0^{+}} \frac{\Phi((1-t) u+t v)+\Phi(u)}{t} \geq 0 .
$$

But

$$
\lim _{t \rightarrow 0^{+}} \frac{\Phi((1-t) u+t v)-\Phi(u)}{t}=\langle D \Phi(u), v-u\rangle .
$$

So we conclude that

$$
\Psi(v)-\Psi(u)-\langle D \Phi(u), v-u\rangle \geq 0
$$

which is the definition of a critical point of $I$. Therefore, $u$ is a critical point of $I$. 
Now, we can prove Corollary 4.1.3.

Proof of Corollary 4.1.3. By assertion (i), $u_{0}$ is a critical point of

$$
I_{K}[u]=\Psi_{K}(u)-\Phi(u)
$$

So,

$$
\Psi_{K}(v)-\Psi_{K}\left(u_{0}\right) \geq\left\langle D \Phi\left(u_{0}\right), v-u_{0}\right\rangle, \quad \forall v \in V
$$

where $\left\langle D \Phi\left(u_{0}\right), v-u_{0}\right\rangle=\int_{\Omega} D \Phi\left(u_{0}\right)\left(v-u_{0}\right) d x$. Equivalently,

$$
\frac{1}{2} \int_{\Omega}|\nabla v|^{2} d x-\frac{1}{2} \int_{\Omega}\left|\nabla u_{0}\right|^{2} d x \geq \int_{\Omega} D \Phi\left(u_{0}\right)\left(v-u_{0}\right) d x, \quad \forall v \in K .
$$

By (ii) of Corollary 4.1.3, there exists $v_{0} \in K$ such that $v_{0}$ satisfies

$$
-\Delta v_{0}=D \Phi\left(u_{0}\right)
$$

in the weak sense, i.e.,

$$
\int_{\Omega} \nabla v_{0} \cdot \nabla w d x=\int_{\Omega} D \Phi\left(u_{0}\right) w d x \quad \forall w \in V
$$

Letting $w=v_{0}-u_{0}$, it follows that

$$
\int_{\Omega} \nabla v_{0} \cdot \nabla\left(v_{0}-u_{0}\right) d x=\int_{\Omega} D \Phi\left(u_{0}\right)\left(v_{0}-u_{0}\right) d x
$$

Thus, we can let $v=v_{0}$ in (4.8) to get,

$$
\begin{aligned}
\frac{1}{2} \int_{\Omega}\left|\nabla v_{0}\right|^{2} d x-\frac{1}{2} \int_{\Omega}\left|\nabla u_{0}\right|^{2} d x & \geq \int_{\Omega} D \Phi\left(u_{0}\right)\left(v_{0}-u_{0}\right) d x \\
& =\int_{\Omega} \nabla v_{0} \cdot \nabla\left(v_{0}-u_{0}\right) d x .
\end{aligned}
$$

Since $\Psi$ is Gâteaux differentiable at $v_{0} \in K$, we have $\partial \Psi\left(v_{0}\right)=\left\{D \Psi\left(v_{0}\right)\right\}$. In addition, $\Psi$ is convex and so we have

$$
\begin{aligned}
\frac{1}{2} \int_{\Omega}\left|\nabla u_{0}\right|^{2} d x-\frac{1}{2} \int_{\Omega}\left|\nabla v_{0}\right|^{2} d x & \geq \int_{\Omega} D \Psi\left(u_{0}\right)\left(u_{0}-v_{0}\right) d x \\
& =\int_{\Omega} \nabla v_{0} \cdot \nabla\left(u_{0}-v_{0}\right) d x
\end{aligned}
$$


Therefore, by (4.9) and (4.10), we get

$$
\frac{1}{2} \int_{\Omega}\left|\nabla v_{0}\right|^{2} d x-\frac{1}{2} \int_{\Omega}\left|\nabla u_{0}\right|^{2} d x=\int_{\Omega} \nabla v_{0} \cdot \nabla\left(v_{0}-u_{0}\right) d x
$$

which implies that

$$
\frac{1}{2} \int_{\Omega}\left|\nabla v_{0}-\nabla u_{0}\right|^{2} d x=0
$$

So $v_{0}=u_{0}$ and thus $u_{0}$ is a solution to the equation

$$
-\Delta u=|u|^{p-2} u+f(x)
$$

To prove that $u_{0}$ is a critical point of $I_{K}[u]$, we need to prove that $I_{K}[\cdot]$ is weakly lower semi-continuous. We will need two lemmas before we begin the proof for $I_{K}[\cdot]$ being weakly lower semi-continuous.

Lemma 4.1.4. Let $V$ be a reflexive Banach space and $\Psi: V \rightarrow(-\infty, \infty]$ be a convex function. If $\partial \Psi(u) \neq \emptyset$ and $u_{n} \rightarrow u$ weakly in $V$, then

$$
\liminf _{n \rightarrow \infty} \Psi\left(u_{n}\right) \geq \Psi(u)
$$

Proof. Let $\left\{u_{n}\right\}_{n=1}^{\infty}$ be a sequence converging to $u$ weakly in V, i.e.,

$$
u_{n} \rightarrow u \quad \text { weakly in } V \text {. }
$$

Let $u^{*} \in \partial \Psi(u)$. Then

$$
\Psi\left(u_{n}\right)-\Psi(u) \geq\left\langle u_{n}-u, u^{*}\right\rangle .
$$

Taking $\liminf \operatorname{in}_{n \rightarrow \infty}$ on both sides, we get

$$
\liminf _{n \rightarrow \infty} \Psi\left(u_{n}\right) \geq \Psi(u)+\lim _{n \rightarrow \infty}\left\langle u_{n}-u, u^{*}\right\rangle .
$$

Since $u_{n}$ converges to $u$ weakly in $V$, i,e.,

$$
\lim _{n \rightarrow \infty}\left\langle u_{n}, u^{*}\right\rangle=\left\langle u, u^{*}\right\rangle
$$


it follows that

$$
\liminf _{n \rightarrow \infty} \Psi\left(u_{n}\right) \geq \Psi(u)
$$

Lemma 4.1.5. Suppose that $\Psi(\cdot)$ is differentiable on $K$. Then $\Psi_{K}(\cdot)$ is weakly lower semi-continuous on $K$.

Proof. Let $u \in K$. We show that $D \Psi(u) \in \partial \Psi_{K}(u)$. If this is true, then it follows by Lemma 4.1 .4 that $\Psi_{K}$ is weakly lower semi-continuous. We need to verify that

$$
\Psi_{K}(v)-\Psi_{K}(u) \geq\langle D \Psi(u), v-u\rangle, \quad \forall v \in V
$$

However, this follows from

$$
\Psi(v)-\Psi(u) \geq\langle D \Psi(u), v-u\rangle, \quad \forall v \in V
$$

by applying the definition of the subdifferential followed by the definition of the restriction of $\Psi$ to $K$.

Now (4.12) is always true since $\Psi(\cdot)$ is convex. Thus $D \Psi(u) \in \partial \Psi_{K}(u)$ and by Lemma 4.1 .4 , we see that $\Psi_{K}(\cdot)$ is weakly lower semi-continuous.

Finally, we come to the following lemma which states that $I_{K}[\cdot]$ is weakly lower semi-continuous.

Lemma 4.1.6. Let $V$ be a reflexive Banach space and let the functional $I_{K}$ : $V \rightarrow(-\infty, \infty]$ be defined by

$$
I_{K}[u]=\Psi_{K}(u)-\Phi(u)
$$

where $\Phi(u)$ and $\Psi_{K}(u)$ are defined by (4.4) and (4.6) respectively. Then $I_{K}[\cdot]$ is weakly lower semi-continuous.

Proof. By Lemma 4.1.4 and Lemma 4.1.5, $\Psi_{K}(\cdot)$ is weakly lower semi-continuous. We only need to prove that $\Phi(\cdot)$ is continuous. Recall that

$$
\Phi(u)=\frac{1}{p} \int_{\Omega}|u|^{p} d x+\int_{\Omega} f u d x .
$$


Let $\left\{u_{n}\right\}_{n=1}^{\infty}$ be a sequence converging to $u$ weakly in $H^{2}(\Omega)$. By the Sobolev Embedding Theorem (see Theorem 2.3.2), $H^{2}(\Omega)$ is compactly embedded in $L^{t}(\Omega)$, i.e.,

$$
H^{2}(\Omega) \hookrightarrow L^{t}(\Omega),
$$

for $2 \leq t<t^{*}$, where $t^{*}=\frac{2 n}{n-4}$ for $n>4$ and $t^{*}=\infty$ for $n \leq 4$. So,

$$
\lim _{n \rightarrow \infty} \int_{\Omega}\left|u_{n}\right|^{p} d x=\int_{\Omega}|u|^{p} d x .
$$

In addition, it follows from the previous argument and the fact that $f \in L^{2}(\Omega)$,

$$
\lim _{n \rightarrow \infty} \int_{\Omega} f u_{n} d x=\int_{\Omega} f u d x .
$$

In other words,

$$
\Phi\left(u_{n}\right) \rightarrow \Phi(u) .
$$

Thus $\Phi(\cdot)$ is continuous.

Finally, we prove that $I_{K}[\cdot]$ is weakly lower semi-continuous. By the definition of $I_{K}[\cdot]$ and taking $\liminf f_{n \rightarrow \infty}$ on both sides, we have

$$
\liminf _{n \rightarrow \infty} I_{K}\left[u_{n}\right]=\liminf _{n \rightarrow \infty}\left(\Psi_{K}\left(u_{n}\right)-\Phi\left(u_{n}\right)\right) .
$$

Since $\Phi(\cdot)$ is continuous, it follows that

$$
\lim _{n \rightarrow \infty} \Phi\left(u_{n}\right)=\Phi(u) .
$$

Also, $\Psi_{K}(\cdot)$ is weakly lower semi-continuous, i.e.,

$$
\Psi_{K}(u) \leq \liminf _{n \rightarrow \infty} \Psi_{K}\left(u_{n}\right) .
$$

Thus, combining (4.13) and (4.14), we obtain

$$
\begin{aligned}
\liminf _{n \rightarrow \infty} I_{K}\left[u_{n}\right] & =\liminf _{n \rightarrow \infty} \Psi_{K}\left(u_{n}\right)-\lim _{n \rightarrow \infty} \Phi\left(u_{n}\right) \\
& \geq \Psi_{K}(u)-\Phi(u) \\
& =I_{K}[u] .
\end{aligned}
$$

Thus, $I_{K}[\cdot]$ is weakly lower semi-continuous. 
Now we can begin to prove our main result stated in the following theorem.

Theorem 4.1.7. Let $2<p<\bar{p}$ where $\bar{p}=\frac{2 n-4}{n-4}$ for $n>4$ and $\bar{p}=\infty$ for $n \leq 4$. Then there exists $\lambda>0$ such that for $\|f\|_{L^{2}(\Omega)}<\lambda$, problem (4.1) has a weak solution $u_{0} \in H^{2}(\Omega) \cap H_{0}^{1}(\Omega)$.

Proof. We apply Corollary 4.1.3 where

$$
\Psi(u)=\frac{1}{2} \int_{\Omega}|\nabla u|^{2} d x, \quad \Phi(u)=\frac{1}{p} \int_{\Omega}|u|^{p} d x+\int_{\Omega} f u d x,
$$

and

$$
K:=K(r)=\left\{u \in V ;\|u\|_{H^{2}(\Omega)} \leq r\right\},
$$

for some $r>0$ to be determined. We begin by proving that there exists $u_{0} \in K$ such that

$$
I_{K}\left[u_{0}\right]=\min _{u \in K} I_{K}[u] .
$$

In other words, there is a $u_{0} \in K$ such that $u_{0}$ is a minimizer and thus, by Proposition 4.1.1, a critical point of $I_{K}[u]$.

Let $m:=\inf _{u \in K} I_{K}[u]$. Note that

$$
m:=\inf _{u \in K} I_{K}[u] \leq I_{K}[0]=0<\infty .
$$

Using Hölder's inequality on $\Phi(u)$, we have that

$$
\begin{aligned}
\Phi(u) & =\frac{1}{p} \int_{\Omega}|u|^{p} d x+\int_{\Omega} f u d x \\
& \leq \frac{1}{p}\|u\|_{L^{p}(\Omega)}^{p}+\|f\|_{L^{2}(\Omega)}\|u\|_{L^{2}(\Omega)} .
\end{aligned}
$$

By the Sobolev Embedding Theorem (see Theorem 2.3.2), the Sobolev space $H^{2}(\Omega)$ is compactly embedded in $L^{t}(\Omega)$ for $t<t^{*}$ where $t^{*}=\frac{2 n}{n-4}$ for $n>4$ and $t^{*}=\infty$ for $n \leq 4$. Thus we get,

$$
\Phi(u) \leq C_{1}\|u\|_{H^{2}(\Omega)}^{p}+C_{2}\|u\|_{H^{2}(\Omega)}
$$


for some positive constants $C_{1}$ and $C_{2}$. Since $\|u\|_{H^{2}(\Omega)} \leq r$ it follows that

$$
\Phi(u) \leq C_{1} r^{p}+C_{2} r<\infty
$$

Since $\Psi(u)$ is nonnegative, we have that

$$
I_{K}[u]:=\Psi_{K}(u)-\Phi(u) \geq \Psi_{K}(u)-\left(C_{1} r^{p}+C_{2} r\right)>-\infty \quad \forall u \in K
$$

So $I_{K}[u]$ is bounded below. Thus we can assume that $m$ is finite. Let $\left\{u_{j}\right\}_{j=1}^{\infty}$ be a minimizing sequence, i.e., $I_{K}\left[u_{j}\right] \rightarrow m$ as $j \rightarrow \infty$. We want to show that $I_{K}[\cdot]$ attains its minimum in $K$. Recall that $\left\{u_{j}\right\}_{k=1}^{\infty} \subset K$ implies $\left\|u_{j}\right\|_{H^{2}(\Omega)} \leq r$, for all $j$. So $\left\{u_{j}\right\}_{j=1}^{\infty}$ has a converging subsequence $\left\{u_{j_{k}}\right\}_{k=1}^{\infty}$ such that $u_{j_{k}} \rightarrow u_{0}$ weakly for some $u_{0} \in H^{2}(\Omega)$. Also $K$ is weakly closed and so $u_{0} \in K$. Since $I_{K}[\cdot]$ is weakly lower semi-continuous by Lemma 4.1.6, it follows that

$$
m \leq I_{K}\left[u_{0}\right] \leq \liminf _{k \rightarrow \infty} I_{K}\left[u_{j_{k}}\right]=m .
$$

So $u_{0}$ is a minimizer and thus by Proposition 4.1.1, $u_{0}$ is a critical point of $I_{K}[u]$. Finally, we verify that $u_{0}$ satisfies the existence condition in Corollary 4.1.3. We need to prove that there exists $v_{0} \in K$ such that

$$
-\Delta v_{0}=\left|u_{0}\right|^{p-2} u_{0}+f(x) .
$$

First, we will show that there exists $v_{0} \in H^{2}(\Omega) \cap H_{0}^{1}(\Omega)$ satisfying

$$
\begin{cases}-\Delta v=\left|u_{0}\right|^{p-2} u_{0}+f(x), & x \in \Omega, \\ v=0, & x \in \partial \Omega .\end{cases}
$$

Consider the Euler-Lagrange functional corresponding to (4.15),

$$
J[v]=\frac{1}{2} \int_{\Omega}|\nabla v|^{2} d x-\int_{\Omega}\left(\left|u_{0}\right|^{p-2} u_{0} v+f v\right) d x, \quad \forall v \in H_{0}^{1}(\Omega) .
$$

Since $J[\cdot]$ is coercive, i.e.,

$$
\lim _{\|v\|_{H^{2}(\Omega)} \rightarrow \infty} J[v]=\infty
$$


and is convex, it admits its minimum at some point $v_{0} \in H^{2}(\Omega) \cap H_{0}^{1}(\Omega)$. So $v_{0}$ satisfies (4.15) in the weak sense. Indeed, let $\phi \in H_{0}^{1}(\Omega)$ and define $g: \mathbb{R} \rightarrow \mathbb{R}$ by

$$
\begin{aligned}
g(t)= & J\left[v_{0}+t \phi\right] \\
= & \frac{1}{2} \int_{\Omega}\left|\nabla v_{0}+t \nabla \phi\right|^{2} d x-\int_{\Omega}\left(\left|u_{0}\right|^{p-2} u_{0} v_{0}+t\left|u_{0}\right|^{p-2} u_{0} \phi+f v_{0}+t f \phi\right) d x \\
= & \frac{1}{2} \int_{\Omega}\left|\nabla v_{0}\right|^{2} d x+t \int_{\Omega} \nabla v_{0} \cdot \nabla \phi d x+\frac{t^{2}}{2} \int_{\Omega}|\nabla \phi|^{2} d x \\
& -\int_{\Omega}\left(\left|u_{0}\right|^{p-2} u_{0} v_{0}+t\left|u_{0}\right|^{p-2} u_{0} \phi+f v_{0}+t f \phi\right) d x .
\end{aligned}
$$

Note that

$$
g(0)=J\left[v_{0}\right] \leq J\left[v_{0}+t \phi\right]=g(t) .
$$

So $g^{\prime}(0)=0$ implies

$$
0=g^{\prime}(0)=\int_{\Omega} \nabla v_{0} \cdot \nabla \phi d x-\int_{\Omega}\left(\left|u_{0}\right|^{p-2} u_{0} \phi+f \phi\right) d x .
$$

Thus

$$
\int_{\Omega} \nabla v_{0} \cdot \nabla \phi d x=\int_{\Omega}\left(\left|u_{0}\right|^{p-2} u_{0}+f\right) \phi d x, \quad \forall \phi \in H_{0}^{1}(\Omega) .
$$

Therefore, $v_{0}$ is a weak solution of (4.15).

Next, we will show that $v_{0} \in K$. In other words, we want to show that $\left\|v_{0}\right\|_{H^{2}(\Omega)} \leq r$. Recall that

$$
K:=K(r)=\left\{u \in H^{2}(\Omega) \cap H_{0}^{1}(\Omega) ;\|u\|_{H^{2}(\Omega)} \leq r\right\},
$$

for some $r>0$ to be determined. By the elliptic regularity theorem (see Theorem 2.4.6), we have that

$$
\begin{aligned}
\left\|v_{0}\right\|_{H^{2}(\Omega)} & \leq C\left(\left\|\left|u_{0}\right|^{p-2} u_{0}\right\|_{L^{2}(\Omega)}+\|f\|_{L^{2}(\Omega)}\right) \\
& =C\left(\left(\int_{\Omega}\left(\left|u_{0}\right|^{p-1}\right)^{2} d x\right)^{\frac{1}{2}}+\|f\|_{L^{2}(\Omega)}\right) .
\end{aligned}
$$

Note that

$$
\left(\int_{\Omega}\left|u_{0}\right|^{2(p-1)} d x\right)^{\frac{1}{2(p-1)}(p-1)}=\left\|u_{0}\right\|_{L^{2(p-1)}(\Omega)}^{p-1} .
$$


Thus

$$
\left\|v_{0}\right\|_{H^{2}(\Omega)} \leq C\left(\left\|u_{0}\right\|_{L^{2(p-1)}(\Omega)}^{p-1}+\|f\|_{L^{2}(\Omega)}\right)
$$

where $C$ is a constant depending on $\Omega$. Using the assumption that $\|f\|_{L^{2}(\Omega)}<\lambda$, for $\lambda>0$ and our convex subset $K$ of $V$, i.e., $\left\|u_{0}\right\|_{H^{2}(\Omega)} \leq r$, we have

$$
\left\|v_{0}\right\|_{H^{2}(\Omega)}<C\left(\left\|u_{0}\right\|_{L^{2(p-1)}(\Omega)}^{p-1}+\lambda\right)
$$

Since $2<2(p-1)<t^{*}$ and $u_{0} \in K$, we have that

$$
\begin{aligned}
\left\|v_{0}\right\|_{H^{2}(\Omega)} & <C_{1}\left(\left\|u_{0}\right\|_{H^{2}(\Omega)}^{p-1}+\lambda\right) \\
& <C_{1}\left(r^{p-1}+\lambda\right) .
\end{aligned}
$$

where $C_{1}$ is a constant in terms of $p$ and $\Omega$. Since $\lambda>0$, we can choose $\lambda$ small enough such that for some fixed $r$,

$$
C_{1}\left(r^{p-1}+\lambda\right) \leq r
$$

So $\left\|v_{0}\right\|_{H^{2}(\Omega)} \leq r$ which implies that $v_{0} \in K(r)$. Since $I_{K}[u]$ has a critical point $u_{0}$ and there exists $v_{0} \in K$ satisfying $-\Delta v_{0}=\left|u_{0}\right|^{p-2} u_{0}+f(x)$, we can conclude by Corollary 4.1.3 that (4.3) has a solution $u_{0} \in H^{2}(\Omega) \cap H_{0}^{1}(\Omega)$.

\subsection{Semilinear Elliptic Equation Involving the Fractional Laplacian}

In this section, we extend our results to the fractional Laplacian operator. Consider the nonlocal semilinear elliptic problem

$$
\begin{cases}(-\Delta)^{s} u=|u|^{p-2} u+f(x), & x \in \Omega, \\ u=0, & x \in \mathbb{R}^{n} \backslash \Omega,\end{cases}
$$


where $s \in(0,1), n>2 s, \Omega$ is an open bounded domain in $\mathbb{R}^{n}$ with $C^{2}$-boundary and $f \in L^{2}(\Omega)$. We begin by providing a definition for $V, \Psi, \Phi$, and $K$ in order to utilize the variational principle given in [17].

Let $V=\mathbb{H}_{0}^{s}(\Omega) \cap L^{p}(\Omega)$ be a Banach space equipped with the norm

$$
\|u\|_{V}:=\|u\|_{\mathbb{H}_{0}^{s}(\Omega)}+\|u\|_{L^{p}(\Omega)}
$$

Consider the convex and weakly closed subset $K$ of $V$ defined by the following two cases:

(i) If $2>\frac{n}{2 s}$, then we have the following convex subset

$$
K_{1}:=K_{1}(r)=\left\{u \in V:\|u\|_{L^{\infty}(\Omega)} \leq r\right\}
$$

(ii) If $\frac{2 n}{n+2 s} \leq 2 \leq \frac{n}{2 s}$, then we have the following convex subset

$$
K_{2}:=K_{2}(r)=\left\{u \in V:\|u\|_{L^{q}(\Omega)} \leq r\right\}
$$

where $q \in\left[2, \frac{2 n}{n-4 s}\right)$,

for some $r>0$ to be determined. We denote the set $K$ to be the sets $K_{1}$ and $K_{2}$ throughout the thesis for simplicity. If we need to refer to a specific case of $K$, only then we will use the notation $K_{1}$ and $K_{2}$ presented above. To see that $K$ is convex and weakly closed, we present the proof of the statement in two lemmas. The first lemma states that $K$ is convex and the second lemma states that $K$ is weakly closed.

Lemma 4.2.1. Let $r>0$. The sets

$$
K_{1}:=K_{1}(r)=\left\{u \in V:\|u\|_{L^{\infty}(\Omega)} \leq r\right\}
$$

and

$$
K_{2}:=K_{2}(r)=\left\{u \in V:\|u\|_{L^{q}(\Omega)} \leq r\right\}
$$

where $q \in\left[2, \frac{2 n}{n-4 s}\right)$ are convex. 
Proof. We only present the proof of the set $K_{1}$ as the set $K_{2}$ can be proved similarly. Let $u_{1}, u_{2} \in K_{1}$. Then

$$
\left\{\begin{array}{l}
\left\|u_{1}\right\|_{L^{\infty}(\Omega)} \leq r \\
\left\|u_{2}\right\|_{L^{\infty}(\Omega)} \leq r .
\end{array}\right.
$$

for some $r>0$ to be determined. In addition, let $0<\lambda<1$. Then

$$
\begin{aligned}
\left\|(1-\lambda) u_{1}+\lambda u_{2}\right\|_{L^{\infty}(\Omega)} & \leq\left\|(1-\lambda) u_{1}\right\|_{L^{\infty}(\Omega)}+\left\|\lambda u_{2}\right\|_{L^{\infty}(\Omega)} \\
& =|1-\lambda|\left\|u_{1}\right\|_{L^{\infty}(\Omega)}+|\lambda|\left\|u_{2}\right\|_{L^{\infty}(\Omega) .}
\end{aligned}
$$

Since $\left\|u_{1}\right\|_{L^{\infty}(\Omega)} \leq r$ and $\left\|u_{2}\right\|_{L^{\infty}(\Omega)} \leq r$, it follows that

$$
\left\|(1-\lambda) u_{1}+\lambda u_{2}\right\|_{L^{\infty}(\Omega)} \leq(1-\lambda) r+\lambda r=r .
$$

Thus, $K_{1}(r)=\left\{u \in V:\|u\|_{L^{\infty}(\Omega)} \leq r\right\}$ is convex.

Lemma 4.2.2. The sets $K_{1}$ and $K_{2}$ defined in Lemma 4.2.1 are weakly closed.

Proof. We proceed by considering two cases.

CASE 1: Let $2>\frac{n}{2 s}$.

Let $\left\{u_{j}\right\}_{j=1}^{\infty}$ be a sequence in $K_{1}(r)$ such that

$$
u_{j} \rightarrow u \text { weakly in } V \text {. }
$$

Then there exists a subsequence of $u_{j}$, denoted by $u_{j}$, such that

$$
u_{j} \rightarrow u \quad \text { a.e. in } \Omega \text {. }
$$

It now follows from

$$
\left|u_{j}(x)\right| \leq\left\|u_{j}\right\|_{L^{\infty}(\Omega)} \leq r
$$

that $|u(x)| \leq r$ for a.e. $x \in \Omega$. Thus, $\|u\|_{L^{\infty}(\Omega)} \leq r$. Therefore, $u \in K_{1}(r)$ and so $K_{1}(r)$ is closed. 
CASE 2: Let $\frac{2 n}{n+2 s} \leq 2 \leq \frac{n}{2 s}$.

Let $\left\{u_{j}\right\}_{j=1}^{\infty}$ be a sequence in $K_{2}(r)$ such that

$$
u_{j} \rightarrow u \text { weakly in } V \text {. }
$$

Then there exists a subsequence of $u_{j}$, denoted by $u_{j}$, such that

$$
u_{j} \rightarrow u \quad \text { a.e. in } \Omega \text {. }
$$

But $\left\|u_{j}\right\|_{L^{q}(\Omega)} \leq r$ and so we can conclude that $\left\{u_{j}\right\}_{j=1}^{\infty}$ is bounded in $L^{q}(\Omega)$. Thus, up to a subsequence, there exists $u \in L^{q}(\Omega)$ such that

$$
u_{j} \rightarrow u \quad \text { weakly in } L^{q}(\Omega)
$$

and by the weak lower semi-continuity of the norm in $L^{q}(\Omega)$, we obtain

$$
\|u\|_{L^{q}(\Omega)} \leq \liminf _{j \rightarrow \infty}\left\|u_{j}\right\|_{L^{q}(\Omega)} \leq r .
$$

Therefore, $u \in K_{2}(r)$ and so $K_{2}(r)$ is closed.

Returning back to the set-up of the problem, let $I: V \rightarrow \mathbb{R}$ be the functional corresponding to (4.16),

$$
I[u]=\frac{1}{2} \int_{\mathbb{R}^{n} \times \mathbb{R}^{n}} \frac{|u(x)-u(y)|^{2}}{|x-y|^{n+2 s}} d x d y-\frac{1}{p} \int_{\Omega}|u|^{p} d x-\int_{\Omega} f u d x .
$$

Define $\Phi: V \rightarrow \mathbb{R}$ by

$$
\Phi(u)=\frac{1}{p} \int_{\Omega}|u|^{p} d x+\int_{\Omega} f u d x,
$$

where $\Phi \in C^{1}(V, \mathbb{R})$ and $\Psi: V \rightarrow \mathbb{R}$ by

$$
\Psi(u)=\frac{1}{2} \int_{\mathbb{R}^{n} \times \mathbb{R}^{n}} \frac{|u(x)-u(y)|^{2}}{|x-y|^{n+2 s}} d x d y,
$$

where $\Psi$ is a proper convex and lower semi-continuous function which is also Gâteaux differentiable on $K$. Define the restriction of $\Psi$ to $K$ by

$$
\Psi_{K}(u)=\left\{\begin{array}{cl}
\frac{1}{2} \int_{\mathbb{R}^{n} \times \mathbb{R}^{n}} \frac{|u(x)-u(y)|^{2}}{|x-y|^{n+2 s}} d x d y, & u \in K, \\
+\infty, & u \notin K .
\end{array}\right.
$$


Finally, we consider the critical points of the functional $I_{K}: V \rightarrow(-\infty, \infty]$ where $I_{K}$ is defined by

$$
I_{K}[u]:=\Psi_{K}(u)-\Phi(u) .
$$

The following corollary is the variational principle specific to our problem.

Corollary 4.2.3. Let $V=\mathbb{H}_{0}^{s}(\Omega) \cap L^{p}(\Omega)$. Let $K$ be a convex and weakly closed subset of $V$. Let $\Phi$ and $\Psi$ be given by (4.17) and (4.18) respectively. Suppose the following two assertions hold:

(i) The functional $I_{K}: V \rightarrow(-\infty, \infty]$ has a critical point $u_{0} \in V$, and;

(ii) there exists $v_{0} \in K$ such that $(-\Delta)^{s} v_{0}=\left|u_{0}\right|^{p-2} u_{0}+f(x)$.

Then $u_{0} \in K$ is a solution of the equation

$$
(-\Delta)^{s} u=|u|^{p-2} u+f(x) .
$$

Proof. By assertion (i), $u_{0}$ is a critical point of

$$
I_{K}[u]=\Psi_{K}(u)-\Phi(u) .
$$

So by Proposition 4.1.1, we get

$$
\Psi_{K}(v)-\Psi_{K}\left(u_{0}\right) \geq\left\langle D \Phi\left(u_{0}\right), v-u_{0}\right\rangle, \quad \forall v \in V,
$$

where $\left\langle D \Phi\left(u_{0}\right), v-u_{0}\right\rangle=\int_{\Omega} D \Phi\left(u_{0}\right)\left(v-u_{0}\right) d x$. In other words,

$$
\begin{array}{r}
\frac{1}{2} \int_{\mathbb{R}^{n} \times \mathbb{R}^{n}} \frac{|v(x)-v(y)|^{2}}{|x-y|^{n+2 s}} d x d y-\frac{1}{2} \int_{\mathbb{R}^{n} \times \mathbb{R}^{n}} \frac{\left|u_{0}(x)-u_{0}(y)\right|^{2}}{|x-y|^{n+2 s}} d x d y \\
\geq \int_{\Omega} D \Phi\left(u_{0}\right)\left(v-u_{0}\right) d x, \quad \forall v \in K .
\end{array}
$$

By assertion (ii), there exists $v_{0} \in K$ such that $v_{0}$ satisfies

$$
(-\Delta)^{s} v_{0}=D \Phi\left(u_{0}\right)
$$


in the weak sense, i.e.,

$$
\int_{\mathbb{R}^{n} \times \mathbb{R}^{n}} \frac{\left(v_{0}(x)-v_{0}(y)\right)(w(x)-w(y))}{|x-y|^{n+2 s}} d x d y=\int_{\Omega} D \Phi\left(u_{0}\right) w d x, \quad \forall w \in V .
$$

Letting $w=v_{0}-u_{0}$, it follows that

$$
\int_{\mathbb{R}^{n} \times \mathbb{R}^{n}} \frac{\left(v_{0}(x)-v_{0}(y)\right)\left(\left(v_{0}-u_{0}\right)(x)-\left(v_{0}-u_{0}\right)(y)\right)}{|x-y|^{n+2 s}} d x d y=\int_{\Omega} D \Phi\left(u_{0}\right)\left(v_{0}-u_{0}\right) d x .
$$

Thus, we can let $v=v_{0}$ in (4.20) to obtain

$$
\begin{gathered}
\frac{1}{2} \int_{\mathbb{R}^{n} \times \mathbb{R}^{n}} \frac{\left|v_{0}(x)-v_{0}(y)\right|^{2}}{|x-y|^{n+2 s}} d x d y-\frac{1}{2} \int_{\mathbb{R}^{n} \times \mathbb{R}^{n}} \frac{\left|u_{0}(x)-u_{0}(y)\right|^{2}}{|x-y|^{n+2 s}} d x d y \\
\geq \int_{\mathbb{R}^{n} \times \mathbb{R}^{n}} \frac{\left(v_{0}(x)-v_{0}(y)\right)\left(\left(v_{0}-u_{0}\right)(x)-\left(v_{0}-u_{0}\right)(y)\right)}{|x-y|^{n+2 s}} d x d y .
\end{gathered}
$$

Since $\Psi$ is Gâteaux differentiable at $v_{0} \in K$, we have $\partial \Psi\left(v_{0}\right)=\left\{D \Psi\left(v_{0}\right)\right\}$. In addition, $\Psi$ is convex and so we have

$$
\begin{gathered}
\frac{1}{2} \int_{\mathbb{R}^{n} \times \mathbb{R}^{n}} \frac{\left|u_{0}(x)-u_{0}(y)\right|^{2}}{|x-y|^{n+2 s}} d x d y-\frac{1}{2} \int_{\mathbb{R}^{n} \times \mathbb{R}^{n}} \frac{\left|v_{0}(x)-v_{0}(y)\right|^{2}}{|x-y|^{n+2 s}} d x d y \\
\geq \int_{\mathbb{R}^{n} \times \mathbb{R}^{n}} \frac{\left(v_{0}(x)-v_{0}(y)\right)\left(\left(v_{0}-u_{0}\right)(x)-\left(v_{0}-u_{0}\right)(y)\right)}{|x-y|^{n+2 s}} d x d y .
\end{gathered}
$$

Therefore, by (4.21) and (4.22), we get

$$
\begin{array}{r}
\frac{1}{2} \int_{\mathbb{R}^{n} \times \mathbb{R}^{n}} \frac{\left|v_{0}(x)-v_{0}(y)\right|^{2}}{|x-y|^{n+2 s}} d x d y-\frac{1}{2} \int_{\mathbb{R}^{n} \times \mathbb{R}^{n}} \frac{\left|u_{0}(x)-u_{0}(y)\right|^{2}}{|x-y|^{n+2 s}} d x d y \\
\quad=\int_{\mathbb{R}^{n} \times \mathbb{R}^{n}} \frac{\left(v_{0}(x)-v_{0}(y)\right)\left(\left(v_{0}-u_{0}\right)(x)-\left(v_{0}-u_{0}\right)(y)\right)}{|x-y|^{n+2 s}} d x d y,
\end{array}
$$

which implies that

$$
\frac{1}{2} \int_{\mathbb{R}^{n} \times \mathbb{R}^{n}} \frac{\left|\left(v_{0}(x)-v_{0}(y)\right)-\left(u_{0}(x)-u_{0}(y)\right)\right|^{2}}{|x-y|^{n+2 s}} d x d y=0 .
$$

So $v_{0}=u_{0}$ and thus $u_{0}$ is a solution to the equation

$$
(-\Delta)^{s} u=|u|^{p-2} u+f(x) .
$$


To prove that $u_{0}$ is a critical point of $I_{K}[u]$, we need to prove that $I_{K}[\cdot]$ is weakly lower semi-continuous. The following lemma states that $I_{K}[\cdot]$ is weakly lower semi-continuous.

Lemma 4.2.4. Let $V$ be a reflexive Banach space and let the functional $I_{K}$ : $V \rightarrow(-\infty, \infty]$ be defined by

$$
I_{K}[u]=\Psi_{K}(u)-\Phi(u)
$$

where $\Phi(u)$ and $\Psi_{K}(u)$ are defined by (4.17) and (4.19) respectively. In addition, let $K_{1}$ and $K_{2}$ be convex and weakly closed subsets of $V$ defined in Lemma 4.2.1. Then $I_{K}[\cdot]$ is weakly lower semi-continuous.

Proof. It can be obtained similarly by the argument presented in Lemma 4.1.6 that $\Psi_{K}(\cdot)$ is weakly lower semi-continuous. Next, we prove that $\Phi(\cdot)$ is continuous. To see this, let $V=\mathbb{H}_{0}^{s}(\Omega) \cap L^{p}(\Omega)$. Recall that

$$
\Phi(u)=\frac{1}{p} \int_{\Omega}|u|^{p} d x+\int_{\Omega} f u d x .
$$

We proceed by considering two cases.

CASE 1: Let $2>\frac{n}{2 s}$.

Let $\left\{u_{n}\right\}_{n=1}^{\infty}$ be a sequence converging to $u$ weakly in $V$. Since $\left\{u_{n}\right\}_{n=1}^{\infty} \subset K$ and $K$ is weakly closed in $V$, it follows that $u \in K$. By Lemma 2.3.9, $\mathbb{H}_{0}^{s}(\Omega)$ is compactly embedded in $L^{2}(\Omega)$. So,

$$
\lim _{n \rightarrow \infty} \int_{\Omega}\left|u_{n}\right|^{2} d x=\int_{\Omega}|u|^{2} d x .
$$

Since $2<p<\infty$, there exists $0<\theta<1$ such that

$$
\frac{1}{p}=\frac{\theta}{2}+\frac{1-\theta}{\infty}
$$

Thus,

$$
\theta=\frac{2}{p}
$$


By the Interpolation Inequality for $L^{p}$-spaces (see Theorem 2.1.12), we get

$$
\|u\|_{L^{p}(\Omega)} \leq\|u\|_{L^{2}(\Omega)}^{\frac{2}{p}}\|u\|_{L^{\infty}(\Omega)}^{\left(1-\frac{2}{p}\right)}
$$

So,

$$
\left\|u_{n}-u\right\|_{L^{p}(\Omega)} \leq\left\|u_{n}-u\right\|_{L^{2}(\Omega)}^{\frac{2}{p}}\left\|u_{n}-u\right\|_{L^{\infty}(\Omega)}^{\left(1-\frac{2}{p}\right)}
$$

But $\left\|u_{n}-u\right\|_{L^{2}(\Omega)}^{\frac{2}{p}} \rightarrow 0$ by compactness and $\left\|u_{n}-u\right\|_{L^{\infty}(\Omega)}^{\left(1-\frac{2}{p}\right)} \leq 2 r^{\left(1-\frac{2}{p}\right)}$ since $u_{n}, u \in$ $K$. Thus,

$$
\left\|u_{n}-u\right\|_{L^{p}(\Omega)} \rightarrow 0
$$

from which we obtain

$$
\frac{1}{p} \int_{\Omega}\left|u_{n}\right|^{p} d x \rightarrow \frac{1}{p} \int_{\Omega}|u|^{p} d x .
$$

It also follows from the previous argument and the fact that $f \in L^{2}(\Omega)$,

$$
\lim _{n \rightarrow \infty} \int_{\Omega} f u_{n} d x=\int_{\Omega} f u d x
$$

In other words,

$$
\Phi\left(u_{n}\right) \rightarrow \Phi(u) .
$$

CASE 2: Let $\frac{2 n}{n+2 s} \leq 2 \leq \frac{n}{2 s}$.

Let $\left\{u_{n}\right\}_{n=1}^{\infty}$ be a sequence converging to $u$ weakly in $V$. Since $\left\{u_{n}\right\}_{n=1}^{\infty} \subset K$ and $K$ is weakly closed in $V$, it follows that $u \in K$. By Lemma 2.3.9, $\mathbb{H}_{0}^{s}(\Omega)$ is compactly embedded in $L^{2}(\Omega)$. So,

$$
\lim _{n \rightarrow \infty} \int_{\Omega}\left|u_{n}\right|^{2} d x=\int_{\Omega}|u|^{2} d x .
$$

Since $2<p<q$, there exists $0<\theta<1$ such that

$$
\frac{1}{p}=\frac{\theta}{2}+\frac{1-\theta}{q} .
$$

By the Interpolation Inequality for $L^{p}$-spaces (see Theorem 2.1.12), we get

$$
\|u\|_{L^{p}(\Omega)} \leq\|u\|_{L^{2}(\Omega)}^{\theta}\|u\|_{L^{q}(\Omega)}^{(1-\theta)} .
$$


So,

$$
\left\|u_{n}-u\right\|_{L^{p}(\Omega)} \leq\left\|u_{n}-u\right\|_{L^{2}(\Omega)}^{\theta}\left\|u_{n}-u\right\|_{L^{q}(\Omega)}^{(1-\theta)} .
$$

But $\left\|u_{n}-u\right\|_{L^{2}(\Omega)}^{\theta} \rightarrow 0$ by compactness and $\left\|u_{n}-u\right\|_{L^{q}(\Omega)}^{(1-\theta)} \leq 2 r^{(1-\theta)}$ since $u_{n}, u \in K$. Thus,

$$
\left\|u_{n}-u\right\|_{L^{p}(\Omega)} \rightarrow 0
$$

from which we obtain

$$
\frac{1}{p} \int_{\Omega}\left|u_{n}\right|^{p} d x \rightarrow \frac{1}{p} \int_{\Omega}|u|^{p} d x .
$$

It also follows from the previous argument and the fact that $f \in L^{2}(\Omega)$,

$$
\lim _{n \rightarrow \infty} \int_{\Omega} f u_{n} d x=\int_{\Omega} f u d x
$$

i.e.,

$$
\Phi\left(u_{n}\right) \rightarrow \Phi(u) .
$$

Thus $\Phi(\cdot)$ is continuous. Finally, we prove that $I_{K}[\cdot]$ is weakly lower semicontinuous. By the definition of $I_{K}[\cdot]$ and taking $\liminf \operatorname{in}_{n \rightarrow \infty}$ on both sides, we have

$$
\liminf _{n \rightarrow \infty} I_{K}\left[u_{n}\right]=\liminf _{n \rightarrow \infty}\left(\Psi_{K}\left(u_{n}\right)-\Phi\left(u_{n}\right)\right) .
$$

Since $\Phi(\cdot)$ is continuous and by $(4.14)$, it follows that

$$
\begin{aligned}
\liminf _{n \rightarrow \infty} I_{K}\left[u_{n}\right] & =\liminf _{n \rightarrow \infty} \Psi_{K}\left(u_{n}\right)-\lim _{n \rightarrow \infty} \Phi\left(u_{n}\right) \\
& \geq \Psi_{K}(u)-\Phi(u) \\
& =I_{K}[u] .
\end{aligned}
$$

Thus, $I_{K}[\cdot]$ is weakly lower semi-continuous.

Now we state and prove the main result of the paper in the following theorem.

Theorem 4.2.5. Let $2<p<\bar{p}$ where $\bar{p}=\frac{2 n-4 s}{n-4 s}$ for $n>4 s$ and $\bar{p}=\infty$ for $n \leq 4 s$. Then there exists $\lambda>0$ such that for $\|f\|_{L^{2}(\Omega)}<\lambda$, problem (4.2) has a weak solution $u_{0} \in \mathbb{H}_{0}^{s}(\Omega) \cap L^{p}(\Omega)$. 
Proof. We apply Corollary 4.2 .3 where

$$
\Psi(u)=\frac{1}{2} \int_{\mathbb{R}^{n} \times \mathbb{R}^{n}} \frac{|u(x)-u(y)|^{2}}{|x-y|^{n+2 s}} d x d y, \quad \Phi(u)=\frac{1}{p} \int_{\Omega}|u|^{p} d x+\int_{\Omega} f u d x
$$

and we present the convex and weakly closed subset $K$ of $V$ in the following two cases:

CASE 1: If $2>\frac{n}{2 s}$, then we have the following convex subset

$$
K_{1}:=K_{1}(r)=\left\{u \in V:\|u\|_{L^{\infty}(\Omega)} \leq r\right\}
$$

for some $r>0$ to be determined and

CASE 2: If $\frac{2 n}{n+2 s} \leq 2 \leq \frac{n}{2 s}$, then we have the following convex subset

$$
K_{2}:=K_{2}(r)=\left\{u \in V:\|u\|_{L^{q}(\Omega)} \leq r\right\},
$$

where $q \in\left[2, \frac{2 n}{n-4 s}\right)$, for some $r>0$ to be determined. We begin by proving that there exists $u_{0} \in K$ such that

$$
I_{K}\left[u_{0}\right]=\min _{u \in K} I_{K}[u]
$$

in other words, there exists $u_{0} \in K$ such that $u_{0}$ is a minimizer and thus a critical point of $I_{K}[u]$. Using Hölder's inequality on $\Phi(u)$, we obtain

$$
\begin{aligned}
\Phi(u) & =\frac{1}{p} \int_{\Omega}|u|^{p} d x+\int_{\Omega} f u d x \\
& \leq \frac{1}{p}\|u\|_{L^{p}(\Omega)}^{p}+\|f\|_{L^{2}(\Omega)}\|u\|_{L^{2}(\Omega)} .
\end{aligned}
$$

CASE 1: Let $2>\frac{n}{2 s}$.

Since $\Omega$ is bounded, we have $L^{\infty}(\Omega) \hookrightarrow L^{p}(\Omega)$. So

$$
\Phi(u) \leq C_{1}\|u\|_{L^{\infty}(\Omega)}^{p}+C_{2}\|u\|_{L^{\infty}(\Omega)},
$$

for some positive constants $C_{1}$ and $C_{2}$. Since $\|u\|_{L^{\infty}(\Omega)} \leq r$, it follows that

$$
\Phi(u) \leq C_{1} r^{p}+C_{2} r<\infty
$$


CASE 2: Let $\frac{2 n}{n+2 s} \leq 2 \leq \frac{n}{2 s}$.

Choose $q$ to be such that $2(p-1) \leq q<\frac{2 n}{n-4 s}$. Since $p>2$, it follows that $p<2(p-1)$ and so by an Embedding Theorem for $L^{p}$ spaces (see Theorem 2.3.1), we have $L^{q}(\Omega) \hookrightarrow L^{p}(\Omega)$ for $1 \leq p \leq \infty$.

Thus,

$$
\Phi(u) \leq C_{1}\|u\|_{L^{q}(\Omega)}^{p}+C_{2}\|u\|_{L^{q}(\Omega)},
$$

for some positive constants $C_{1}$ and $C_{2}$. Since $\|u\|_{L^{q}(\Omega)} \leq r$, it follows that

$$
\Phi(u) \leq C_{1} r^{p}+C_{2} r<\infty .
$$

In both cases, we end up with $\Phi(u) \leq C_{1} r^{p}+C_{2} r<\infty$. Since $\Psi(u)$ is nonnegative, we have

$$
I_{K}[u]:=\Psi_{K}(u)-\Phi(u) \geq \Psi_{K}(u)-\left(C_{1} r^{p}+C_{2} r\right)>-\infty, \quad \forall u \in K .
$$

Thus $\inf _{u \in K} I_{K}[u]>-\infty$. Now consider the minimizing sequence $\left\{u_{j}\right\}_{j=1}^{\infty}$ for $I_{K}[\cdot]$, i.e.,

$$
I_{K}\left[u_{j}\right] \rightarrow \inf _{u \in K} I_{K}[u]
$$

as $j \rightarrow \infty$. We want to show that $I_{K}[\cdot]$ attains its minimum in $K$. Note that $\left\{u_{j}\right\}_{j=1}^{\infty} \subset K$ implies that if $2>\frac{n}{2 s}$, then

$$
\left\|u_{j}\right\|_{L^{\infty}(\Omega)} \leq r
$$

for all $j$ and if $\frac{2 n}{n+2 s} \leq 2 \leq \frac{n}{2 s}$, then

$$
\left\|u_{j}\right\|_{L^{q}(\Omega)} \leq r
$$

for all $j$. Now we claim that $\left\{u_{j}\right\}_{j=1}^{\infty}$ is bounded in $\mathbb{H}_{0}^{s}(\Omega) \cap L^{p}(\Omega)$. Indeed,

$$
I_{K}\left[u_{j}\right]=\frac{1}{2}\left\|u_{j}\right\|_{\mathbb{H}_{0}^{s}(\Omega)}^{2}-\Phi\left(u_{j}\right) \rightarrow m \in \mathbb{R}
$$


Since $\Phi\left(u_{j}\right)$ is bounded (see (4.23) and (4.24)) and $I_{K}\left[u_{j}\right] \rightarrow m$, it follows that $\left\|u_{j}\right\|_{\mathbb{H}_{0}^{s}(\Omega)}$ is bounded. Therefore, there exists a subsequence of $\left\{u_{j}\right\}_{j=1}^{\infty}$, call it $\left\{u_{j}\right\}_{j=1}^{\infty}$ again such that

$$
u_{j} \rightarrow u_{0} \quad \text { weakly in } \mathbb{H}_{0}^{s}(\Omega)
$$

But by Lemma 2.3.9, the embedding $\mathbb{H}_{0}^{s}(\Omega) \hookrightarrow L^{t}(\Omega)$ is compact for any $t \in$ $\left[1,2_{s}^{*}\right)$, so

$$
u_{j} \rightarrow u_{0} \quad \text { strongly in } L^{t}(\Omega) .
$$

Note that $u_{0} \in \mathbb{H}_{0}^{s}(\Omega) \cap L^{t}(\Omega)$ implies $u_{0} \in \mathbb{H}_{0}^{s}(\Omega) \cap L^{p}(\Omega)$ and $K$ is weakly closed, so $u_{0} \in K$. Since $I[\cdot]$ is weakly lower semi-continuous by Lemma 4.2.4, it follows that

$$
\inf _{u \in K} I_{K}[u] \leq I_{K}\left[u_{0}\right] \leq \liminf _{j \rightarrow \infty} I_{K}\left[u_{j}\right]=\inf _{u \in K} I_{K}[u] .
$$

So $u_{0}$ is a minimizer and thus by Proposition 4.1.1, $u_{0}$ is a critical point of $I_{K}[u]$. Finally, we verify that $u_{0}$ satisfies the existence condition in Corollary 4.2.3. We need to prove that there exists $v_{0} \in K$ such that

$$
(-\Delta)^{s} v_{0}=\left|u_{0}\right|^{p-2} u_{0}+f(x) .
$$

We begin by showing that there exists $v_{0} \in \mathbb{H}_{0}^{s}(\Omega) \cap L^{p}(\Omega)$ satisfying

$$
\begin{cases}(-\Delta)^{s} v=\left|u_{0}\right|^{p-2} u_{0}+f(x), & x \in \Omega, \\ v=0, & x \in \mathbb{R}^{n} \backslash \Omega .\end{cases}
$$

Consider the functional corresponding to (4.25),

$J[v]=\frac{1}{2} \int_{\mathbb{R}^{n} \times \mathbb{R}^{n}} \frac{|v(x)-v(y)|^{2}}{|x-y|^{n+2 s}} d x d y-\int_{\Omega}\left(\left|u_{0}\right|^{p-2} u_{0} v+f(x) v\right) d x, \quad \forall v \in \mathbb{H}_{0}^{s}(\Omega)$.

Since $J[\cdot]$ is coercive and convex, it admits its minimum at some point $v_{0} \in$ $\mathbb{H}_{0}^{s}(\Omega)$. So $v_{0}$ satisfies (4.25) in the weak sense. Indeed, let $\phi \in \mathbb{H}_{0}^{s}(\Omega)$ and define 


$$
\begin{aligned}
g: \mathbb{R} \rightarrow & \mathbb{R} \text { by } \\
g(t)= & J\left[v_{0}+t \phi\right] \\
= & \frac{1}{2} \int_{\mathbb{R}^{n} \times \mathbb{R}^{n}} \frac{\left|v_{0}(x)+t \phi(x)-\left(v_{0}(y)+t \phi(y)\right)\right|^{2}}{|x-y|^{n+2 s}} d x d y \\
& -\int_{\Omega}\left(\left|u_{0}\right|^{p-2} u_{0} v+t\left|u_{0}\right|^{p-2} u_{0} \phi+f v+t f \phi\right) d x \\
= & \frac{1}{2} \int_{\mathbb{R}^{n} \times \mathbb{R}^{n}} \frac{\left|v_{0}(x)\right|^{2}+2 v_{0}(x) \cdot t \phi(x)-2 v_{0}(x) \cdot v_{0}(y)-2 v_{0}(x) \cdot t \phi(y)}{|x-y|^{n+2 s}} d x d y \\
& +\frac{1}{2} \int_{\mathbb{R}^{n} \times \mathbb{R}^{n}} \frac{t^{2}|\phi(x)|^{2}-2 t \phi(x) \cdot v(y)-2 t^{2} \phi(x) \cdot \phi(y)}{|x-y|^{n+2 s}} d x d y \\
& +\frac{1}{2} \int_{\mathbb{R}^{n} \times \mathbb{R}^{n}} \frac{\left|v_{0}(y)\right|^{2}+2 t v_{0}(y) \cdot \phi(y)+t^{2}|\phi(y)|^{2}}{|x-y|^{n+2 s}} d x d y \\
& -\int_{\Omega}\left(\left|u_{0}\right|^{p-2} u_{0} v+t\left|u_{0}\right|^{p-2} u_{0} \phi+f v_{0}+t f \phi\right) d x .
\end{aligned}
$$

Note that

$$
g(0)=J\left[v_{0}\right] \leq J\left[v_{0}+t \phi\right]=g(t)
$$

So $g^{\prime}(0)=0$ implies

$$
\begin{aligned}
0=g^{\prime}(0)= & \int_{\mathbb{R}^{n} \times \mathbb{R}^{n}} \frac{\left(v_{0}(x) \cdot \phi(x)\right)-\left(v_{0}(x) \cdot \phi(y)\right)-\left(\phi(x) \cdot v_{0}(y)\right)+\left(v_{0}(y) \cdot \phi(y)\right)}{|x-y|^{n+2 s}} d x d y \\
& -\int_{\Omega}\left(\left|u_{0}\right|^{p-2} u_{0} \phi+f \phi\right) d x .
\end{aligned}
$$

Thus

$$
\int_{\mathbb{R}^{n} \times \mathbb{R}^{n}} \frac{\left(v_{0}(x)-v_{0}(y)\right)(\phi(x)-\phi(y))}{|x-y|^{n+2 s}} d x d y=\int_{\Omega}\left(\left|u_{0}\right|^{p-2} u_{0}+f\right) \phi d x, \quad \forall \phi \in \mathbb{H}_{0}^{s}(\Omega) .
$$

Therefore, $v_{0}$ is a weak solution of (4.25).

Next, we will show that $v_{0} \in K$. In other words, we want to show that if $2>\frac{n}{2 s}$, then

$$
\left\|v_{0}\right\|_{L^{\infty}(\Omega)} \leq r
$$

and if $\frac{2 n}{n+2 s} \leq 2 \leq \frac{n}{2 s}$, then

$$
\left\|v_{0}\right\|_{L^{q}(\Omega)} \leq r .
$$


We prove this by cases, the first being the case where $2>\frac{n}{2 s}$ and the second being the case where $\frac{2 n}{n+2 s} \leq 2 \leq \frac{n}{2 s}$.

CASE 1: Let $2>\frac{n}{2 s}$.

By Lemma 2.4.7 (i) with $t=2$, we have

$$
\begin{aligned}
\left\|v_{0}\right\|_{L^{\infty}(\Omega)} & \leq C\left(\left\|\left|u_{0}\right|^{p-2} u_{0}\right\|_{L^{2}(\Omega)}+\|f\|_{L^{2}(\Omega)}\right) \\
& =C\left(\left(\int_{\Omega}\left(\left|u_{0}\right|^{p-1}\right)^{2} d x\right)^{\frac{1}{2}}+\|f\|_{L^{2}(\Omega)}\right)
\end{aligned}
$$

Note that

$$
\left(\int_{\Omega}\left|u_{0}\right|^{2(p-1)} d x\right)^{\frac{1}{2(p-1)}(p-1)}=\left\|u_{0}\right\|_{L^{2(p-1)}(\Omega)}^{p-1} .
$$

Thus

$$
\left\|v_{0}\right\|_{L^{\infty}(\Omega)} \leq C\left(\left\|u_{0}\right\|_{L^{2(p-1)}(\Omega)}^{p-1}+\|f\|_{L^{2}(\Omega)}\right)
$$

where $C$ is a constant depending on $\Omega$. Utilizing the assumption that $\|f\|_{L^{2}(\Omega)}<\lambda$ for $\lambda>0$, we have

$$
\left\|v_{0}\right\|_{L^{\infty}(\Omega)}<C\left(\left\|u_{0}\right\|_{L^{2(p-1)}(\Omega)}^{p-1}+\lambda\right) .
$$

Since $2<2(p-1)<\infty$ and $u_{0} \in K_{1}$, we obtain

$$
\begin{aligned}
\left\|v_{0}\right\|_{L^{\infty}(\Omega)} & <C_{1}\left(\left\|u_{0}\right\|_{L^{\infty}(\Omega)}^{p-1}+\lambda\right) \\
& <C_{1}\left(r^{p-1}+\lambda\right)
\end{aligned}
$$

where $C_{1}$ is a constant in terms of $p$ and $\Omega$. Since $\lambda>0$, we can choose $\lambda$ small enough such that for some fixed $r$,

$$
C_{1}\left(r^{p-1}+\lambda\right) \leq r
$$

So $\left\|v_{0}\right\|_{L^{\infty}(\Omega)} \leq r$ which implies that $v_{0} \in K_{1}(r)$.

CASE 2: Let $\frac{2 n}{n+2 s} \leq 2 \leq \frac{n}{2 s}$. 
By Lemma 2.4.7 (ii) with $t=2$, we have

$$
\begin{aligned}
\left\|v_{0}\right\|_{L^{q}(\Omega)} & \leq C\left(\left\|\left|u_{0}\right|^{p-2} u_{0}\right\|_{L^{2}(\Omega)}+\|f\|_{L^{2}(\Omega)}\right) \\
& =C\left(\left(\int_{\Omega}\left(\left|u_{0}\right|^{p-1}\right)^{2} d x\right)^{\frac{1}{2}}+\|f\|_{L^{2}(\Omega)}\right) \\
& =C\left(\left\|u_{0}\right\|_{L^{2(p-1)}(\Omega)}^{p-1}+\|f\|_{L^{2}(\Omega)}\right)
\end{aligned}
$$

where $C$ is a constant depending on $\Omega$. Since $\|f\|_{L^{2}(\Omega)}<\lambda$ for $\lambda>0$, we have

$$
\left\|v_{0}\right\|_{L^{q}(\Omega)}<C\left(\left\|u_{0}\right\|_{L^{2(p-1)}(\Omega)}^{p-1}+\lambda\right) .
$$

In addition, $2<2(p-1)<q$ for $q \in\left[2, \frac{2 n}{n-4 s}\right)$ and $u_{0} \in K_{2}$, so it follows that

$$
\begin{aligned}
\left\|v_{0}\right\|_{L^{q}(\Omega)} & <C_{2}\left(\left\|u_{0}\right\|_{L^{q}(\Omega)}^{p-1}+\lambda\right) \\
& <C_{2}\left(r^{p-1}+\lambda\right)
\end{aligned}
$$

where $C_{2}$ is a constant in terms of $p$ and $\Omega$. Following the argument presented in Case 1, we conclude that $v_{0} \in K_{2}(r)$.

Since $I_{K}[u]$ has a critical point $u_{0}$ and there exists $v_{0} \in K$ satisfying $(-\Delta)^{s} v_{0}=$ $\left|u_{0}\right|^{p-2} u_{0}+f(x)$, we can conclude by Corollary 4.2.3 that (4.16) has a solution $u_{0} \in \mathbb{H}_{0}^{s}(\Omega) \cap L^{p}(\Omega)$.

To summarize the thesis, we looked into local and nonlocal semilinear elliptic problems where the nonlinear term is supercritical by means of Sobolev and fractional Sobolev spaces. We applied a new variational principle arising from [17] which deals with problems that lack compactness and resulted the existence of a weak solution to our local and nonlocal problem. We can further extend our results by relaxing the condition on $f \in L^{2}(\Omega)$ to $f \in L^{d}(\Omega)$ where $d>2$. Using the same approach made in Theorem 4.1.7 and Theorem 4.2.5, we can prove the existence of a weak solution to our local and nonlocal problem provided that $\|f\|_{L^{d}(\Omega)}$ is small enough. 


\section{Bibliography}

[1] R.A. Adams and J.J.F Fournier, Sobolev Spaces. Second edition. Pure and Applied Mathematics (Amsterdam), 140. Elsevier/Academic Press, Amsterdam, 2003.

[2] A. Ambrosetti, P.H. Rabinowitz, Dual variational methods in critical point theory and applications. J. Funct. Anal. 14 (1973), 349-381.

[3] F.V. Atkinson, H. Brezis, L.A. Peletier, Nodal solutions of elliptic equations with critical Sobolev exponents. J. Differential Equations 85 (1990), no. 1, 151-170.

[4] A. Bahri, Topological results on a certain class of functionals and applications. J. Funct. Anal. 41 (1981) 397-427.

[5] A. Bahri, J.M. Coron, On a nonlinear elliptic equation involving the Sobolev exponent: the effect of the topology of the domain. Comm. Pure Appl. Math. 41 (1988), 253-294.

[6] U. Biccari, M. Warma, E. Zuazua, Local elliptic regularity for the Dirichlet fractional Laplacian. Adv. Nonlinear Stud. 17 (2017), no. 4, 837-839.

[7] H. Brezis, Functional Analysis, Sobolev Spaces, and Partial Differential Equations. Springer, New York, 2011. 
[8] C. Bucur, E. Valdinoci, Nonlocal diffusion and applications. Lecture Notes of the Unione Matematica Italiana, 20. Springer, [Cham]; Unione Matematica Italiana, Bologna, (2016).

[9] X. Chang, Z. Wang, Nodal and multiple solutions of nonlinear problems involving the fractional Laplacian. J. Differential Equations 256 (2014), no. 8, 2965-2992.

[10] E.N. Dancer, A note on an equation with critical exponent. Bull. London Math. Soc. 20 (1988), 600-602.

[11] E. Di Nezza, G. Palatucci, E. Valdinoci, Hitchhiker's guide to the fractional Sobolev spaces. Bull. Sci. Math. 136 (2012), no. 5, 521-573.

[12] I. Ekeland, R. Temam, Convex Analysis and Variational Problems. American Elsevier Publishing Co., Inc., New York. 1976.

[13] L. C. Evans, Partial Differential Equations, AMS, (1998).

[14] D. Gilbarg, N.S. Trudinger, Elliptic partial differential equations of second order. Second Edition. Reprint of the (1998) edition. Classics in Mathematics. Springer-Verlag, Berlin, (2001).

[15] G. Molica Bisci, V. D. Radulescu, R. Servadei, Variational Methods for Nonlocal Fractional Problems. Encyclopedia of Mathematics and its Applications, 162. Cambridge University Press, Cambridge, 2016.

[16] J. Kazdan, F. Warner, Remarks on some quasilinear elliptic equations. Comm. Pure Appl. Math. 28 (1975), 567-597.

[17] A. Moameni, A variational principle for problems with a hint of convexity. C. R. Math. Acad. Sci. Paris 355 (2017), no. 12, 1236-1241. 
[18] S. Mosconi, N. Shioji, M. Squassina, Nonlocal problems at critical growth in contractible domains. Asymptot. Anal. 95 (2015), no. 1-2, 79-100.

[19] D. Passaseo, Nontrivial solutions of elliptic equations with supercritical exponent in contractible domains. Duke Math. J. 92 (1998), no. 2, 429-457.

[20] D. Passaseo, Multiplicity of nodal solutions for elliptic equations with supercritical exponent in contractible domains. Topol. Methods Nonlinear Anal. 8 (1996), no. 2, 245-262.

[21] D. Passaseo, The effect of the domain shape on the existence of positive solutions of the equation $\Delta u+u^{2^{*}-1}=0$. Topol. Methods Nonlinear Anal. 3 (1994), no. 1, 27-54.

[22] D. Passaseo, Multiplicity of positive solutions of nonlinear elliptic equations with critical Sobolev exponent in some contractible domains. Manuscripta Math. 65 (1989), no. 2, 147-165.

[23] S.I. Pohozaev, Eigenfunctions of the equation $\Delta u+\lambda f(u)=0$. Sov. Math. Dokl. 6 (1965), 1408-1411.

[24] P. H. Rabinowitz, Minimax methods in Critical Point Theory with Applications to Differential Equations. CBMS Regional Conference Series Math. 65, Amer. Math. Soc., Providence (1986).

[25] R.T. Rockafellar, Convex analysis. Princeton Mathematical Series, No. 28 Princeton University Press, Princeton, N.J. (1970).

[26] X. Ros-Oton, J. Serra, The Pohozaev identity for the fractional Laplacian. Arch. Ration. Mech. Anal. 213 (2014), no. 2, 587-628.

[27] S. Secchi, N. Shioji, M. Squassina, Coron problem for fractional equations. Differential Integral Equations 28 (2015), no. 1-2, 103-118. 
[28] R. Servadei, Infinitely many solutions for fractional Laplace equations with subcritical nonlinearity. Recent trends in nonlinear partial differential equations. II. Stationary problems, 317-340, Contemp. Math., 595, Amer. Math. Soc., Providence, RI, (2013).

[29] R. Servadei, E. Valdinoci, Fractional Laplacian equations with critical Sobolev exponent. Rev. Mat. Complut. 28 (2015). no.3, 655-676.

[30] R. Servadei, E. Valdinoci, Mountain pass solutions for non-local elliptic operators. J. Math. Anal. Appl. 389 (2012), no. 2, 887-898.

[31] A. Szulkin, Minimax principles for lower semicontinuous functions and applications to nonlinear boundary value problems. Ann. Inst. H. Poincaré Anal. Non Linéaire 3 (1986), no. 2, 77-109.

[32] G. Tarantello, On nonhomogeneous elliptic equations involving critical Sobolev exponent. Ann. Inst. H. Poincaré Anal. Non Linéaire 9 (1992), no. 3, 281-304.

[33] Y. Wei, X. Su, Multiplicity of solutions for non-local elliptic equations driven by the fractional Laplacian. Calc. Var. Partial Differential Equations 52 (2015), no. 1-2, 95-124.

[34] M.W. Wong, An Introduction to Pseudo-Differential Operators. Third edition. Series on Analysis, Applications and Computation, 6. World Scientific Publishing Co. Pte. Ltd., Hackensack, NJ, 2014.

[35] K. Yosida, Functional Analysis. 5th ed., Berlin-Heidelberg-New York: Springer-Verlag (1978). 